\title{
An analogue of Liouville's Theorem and an application to cubic surfaces
}

\author{
David McKinnon $^{1}$ (D) Mike Roth $^{2}$
}

Received: 18 April 2016 / Revised: 20 June 2016 / Accepted: 4 July 2016 / Published online: 2 August 2016 (C) Springer International Publishing AG 2016

\begin{abstract}
We prove a strong analogue of Liouville's Theorem in Diophantine approximation for points on arbitrary algebraic varieties. We use this theorem to prove a conjecture of the first author for cubic surfaces in $\mathbb{P}^{3}$.
\end{abstract}

Keywords Seshadri constants · Diophantine approximation · Rational points

Mathematics Subject Classification $14 \mathrm{G} 05 \cdot 14 \mathrm{G} 40$

\section{Introduction}

The famous theorem of K.F. Roth (see for example [5, Part D]) gives a sharp upper bound on how well an irrational algebraic number can be approximated by rational numbers. In [10], the authors prove an analogue of Roth's Theorem for algebraic points on arbitrary algebraic varieties. In this paper we generalize, in the sense of [10], Liouville's Approximation Theorem to arbitrary varieties, as well as giving an extension involving the stable base locus.

The research of David McKinnon and Mike Roth was partially supported by NSERC research grants.

$\bowtie$ David McKinnon

dmckinnon@uwaterloo.ca

Mike Roth

mikeroth@mast.queensu.ca

1 Department of Pure Mathematics, University of Waterloo, 200 University Ave. West, Waterloo, ON N2L 3G1, Canada

2 Department of Mathematics and Statistics, Queens University, Jeffery Hall, University Ave., Kingston, ON K7L 3N6, Canada 
The point of view of [10] is that Roth's and Liouville's Theorems are examples of "local Bombieri-Lang phenomena" whereby local positivity of a line bundle influences local accumulation of rational points. Specifically, given a variety $X$, an algebraic point $x \in X$, and an ample line bundle $L$ on $X$, these theorems are expressed as inequalities between $\epsilon_{x}(L)$, the Seshadri constant, measuring local positivity of $L$ near $x$, and $\alpha_{x}(L)$, an invariant measuring how well we can approximate $x$ by rational points.

Roth's Theorem is usually thought of as stronger than Liouville's, but if the locus being approximated is defined over the ground field, Liouville's Theorem is strictly better. On $\mathbb{P}^{1}$ one gains a factor of two. For arbitrary varieties, however, moving past the Seshadri constant into the non-nef part of the big cone can provide even larger gains. We need this improvement for our application in Sect. 4 where we verify a conjecture of the first author for cubic surfaces in $\mathbb{P}^{3}$.

In Sect. 2 we review the definitions and elementary properties of $\alpha_{x}$ and $\epsilon_{x}$. In Sect. 3 we prove the generalized Liouville Theorem (Theorem 3.3). We close the paper in Sect. 4 by computing $\alpha_{x}$ and $\epsilon_{x}$ for an arbitrary nef line bundle and rational point, not on a line, on a smooth cubic surface (where the lines are also rational); we then use this to verify [9, Conjecture 3.2].

\section{Elementary properties of $\alpha$ and $\epsilon$}

In this section, we give a brief overview of the properties of $\alpha$ and $\epsilon$ used in this paper. For a more detailed discussion of $\alpha$, see [10]. For a more detailed discussion of $\epsilon$, there are many good references-see for example [7, Chapter 5]. Proofs of all of the facts listed below can be found in [10].

Let $k$ be a number field, and $X$ a projective variety over $\operatorname{Spec}(k)$ (i.e., a reduced subscheme of some finite-dimensional projective space $\mathbb{P}_{k}^{r}$ ).

The constant $\alpha_{x}$. In order to motivate the definition of $\alpha_{x}$ it is helpful to recall the classical case of approximation on the line. For a point $x \in \mathbb{R}$ the approximation exponent $\tau_{x}$ of $x$ is the unique extended real number $\tau_{x} \in(0, \infty]$ such that the inequality

$$
\left|x-\frac{a}{b}\right| \leqslant \frac{1}{b^{\tau_{x}+\delta}}
$$

has only finitely many solutions $a / b \in \mathbb{Q}$ whenever $\delta>0$ (respectively has infinitely solutions $a / b \in \mathbb{Q}$ whenever $\delta<0$ ). The approximation exponent measures a certain tension between our ability to closely approximate $x$ by rational numbers (the distance term $|x-a / b|$ ) and the complexity (the $1 / b$ term) of the number required to make this approximation. In this notation the 1844 theorem of Liouville [8] is that $\tau_{x} \leqslant d$ for $x \in \mathbb{R}$ algebraic of degree $d$ over $\mathbb{Q}$.

To generalize $\tau_{x}$ to arbitrary projective varieties over $\operatorname{Spec}(k)$ for a number field $k$, we replace the function $|x-a / b|$ by a distance function $d_{v}(x, \cdot)$ depending on a place $v$ of $k$, and measure the complexity of a point via a height function $H_{L}(\cdot)$ depending on an ample line bundle $L$. For an introduction to the theory of heights the reader is referred to any one of [2, Chapter 2], [5, Part B], [6, Chapter III], or [12, Chapter 2]. 
Unless otherwise specified all height functions in this paper are multiplicative, relative to $k$, and come from line bundles on $X$ defined over $k$. In this paper we use the following normalizations. The absolute values are normalized with respect to $k$ : if $v$ is a finite place of $k, \pi$ a uniformizer of the corresponding maximal ideal, and $\kappa$ the residue field then $\|\pi\|_{v}=1 / \# \kappa$; if $v$ is an infinite place corresponding to an embedding $i: k \hookrightarrow \mathbb{C}$ then $\|x\|_{v}=\|i(x)\|^{m_{v}}$ for all $x \in k$, where $m_{v}=1$ or 2 depending on whether $v$ is real or complex. The heights are then normalized so that for a point $x=\left[x_{0}: \cdots: x_{n}\right] \in \mathbb{P}^{n}(k)$, the height with respect to $\mathcal{O}_{\mathbb{P}^{n}}(1)$ is

$$
H(x)=\prod_{v} \max \left(\left\|x_{0}\right\|_{v}, \ldots,\left\|x_{n}\right\|_{v}\right)
$$

where the product ranges over all the places $v$ of $k$.

In order to define a distance function we fix a place $v$ of $k$ and extension (which we also call $v$ ) to $\bar{k}$.

If $v$ is archimedean. We choose a distance function on $X(\bar{k})$ by choosing an embed$\operatorname{ding} X \hookrightarrow \mathbb{P}_{k}^{r}$ and pulling back (via $v$ ) the function on $\mathbb{P}^{r}(\mathbb{C}) \times \mathbb{P}^{r}(\mathbb{C})$ given by the formula

$$
d_{v}(x, y)=\left(1-\frac{\left|\sum_{i=0}^{r} x_{i} \overline{y_{i}}\right|^{2}}{\left(\sum_{i=0}^{r}\left|x_{i}\right|^{2}\right)\left(\sum_{j=0}^{r}\left|y_{j}\right|^{2}\right)}\right)^{\left[k_{v}: \mathbb{R}\right] / 2}
$$

where $x=\left[x_{0}: \cdots: x_{r}\right]$ and $y=\left[y_{0}: \cdots: y_{r}\right]$ are points of $\mathbb{P}^{r}(\mathbb{C})$, and $|\cdot|$ is the absolute value on $\mathbb{C}$ extending the usual absolute value on $\mathbb{R}$, i.e., such that $\mid 3+$ $4 \sqrt{-1} \mid=5$.

If $v$ is non-archimedean. We choose a distance function on $X(\bar{k})$ by choosing an embedding $X \hookrightarrow \mathbb{P}_{k}^{r}$ and pulling back the distance function on $\mathbb{P}^{r}(\bar{k})$ given by the formula

$$
d_{v}(x, y)=\frac{\max _{0 \leqslant i<j \leqslant r}\left(\left\|x_{i} y_{j}-x_{j} y_{i}\right\|_{v}\right)}{\max _{0 \leqslant i \leqslant r}\left(\left\|x_{i}\right\|_{v}\right) \max _{0 \leqslant j \leqslant r}\left(\left\|y_{j}\right\|_{v}\right)}
$$

where $x=\left[x_{0}: \cdots: x_{r}\right]$ and $y=\left[y_{0}: \cdots: y_{r}\right]$ are points of $\mathbb{P}^{r}(\bar{k})$.

These definitions are standard in Arakelov theory, albeit here we have normalized with respect to $k$ rather than $\mathbb{Q}$. (See for instance [2, Section 2.8] where a distance function $\delta_{v}(\cdot, \cdot)$ is defined for each place $v$; the distance functions are related by $\left.d_{v}(\cdot, \cdot)=\delta_{v}(\cdot, \cdot)^{[k: \mathbb{Q}]}.\right)$

Two real valued functions $g$ and $g^{\prime}$ with the same domain are called equivalent if there are positive constants $c \leqslant C$ such that $c g \leqslant g^{\prime} \leqslant C g$ for all values of the domain. The distance functions defined above depend on the choice of embedding into projective space, but by [10, Proposition 2.4] any two embeddings give equivalent distance functions on $X\left(k_{v}\right) \times X\left(k_{v}\right)$. (We may restrict ourselves to points of $X\left(k_{v}\right)$ since any point of $X(\bar{k})$ that does not lie in $X\left(k_{v}\right)$ cannot be approximated by $k$-rational points; see also Remark 2.7.) It follows from the definition of $\alpha_{x}$ below that equivalent distance functions produce the same value of $\alpha_{x}$; thus our definition of $\alpha_{x}$ does not 
depend on the projective embedding chosen to define $d_{v}$. A more geometric definition of distance, and a proof that it is equivalant to the distance formulae above, may be found in Sect. 6.

Definition 2.1 Let $X$ be a projective variety, $x \in X(\bar{k}), L$ a line bundle on $X$. For any sequence $\left\{x_{i}\right\} \subset X(k)$ of distinct points with $d_{v}\left(x, x_{i}\right) \rightarrow 0$ (which we denote by $\left.\left\{x_{i}\right\} \rightarrow x\right)$, we set

$$
A\left(\left\{x_{i}\right\}, L\right)=\left\{\gamma \in \mathbb{R}: d_{v}\left(x, x_{i}\right)^{\gamma} H_{L}\left(x_{i}\right) \text { is bounded from above }\right\} .
$$

Remark 2.2 (a) It follows easily from the definition that if $A\left(\left\{x_{i}\right\}, L\right)$ is nonempty then it is an interval unbounded to the right, i.e., if $\gamma \in A\left(\left\{x_{i}\right\}, L\right)$ then $\gamma+\delta \in A\left(\left\{x_{i}\right\}, L\right)$ for any $\delta>0$.

(b) If $\left\{x_{i}^{\prime}\right\}$ is a subsequence of $\left\{x_{i}\right\}$ then $A\left(\left\{x_{i}\right\}, L\right) \subseteq A\left(\left\{x_{i}^{\prime}\right\}, L\right)$.

Definition 2.3 If $A\left(\left\{x_{i}\right\}, L\right)$ is empty we set $\alpha_{x}\left(\left\{x_{i}\right\}, L\right)=\infty$. Otherwise we set $\alpha_{x}\left(\left\{x_{i}\right\}, L\right)$ to be the infimum of $A\left(\left\{x_{i}\right\}, L\right)$. We call $\alpha_{x}\left(\left\{x_{i}\right\}, L\right)$ the approximation constant of $\left\{x_{i}\right\}$ with respect to $L$.

As $i \rightarrow \infty$ we have $d_{v}\left(x, x_{i}\right) \rightarrow 0$. We thus expect that $d_{v}\left(x, x_{i}\right)^{\gamma} H_{L}\left(x_{i}\right)$ goes to 0 for large $\gamma$ and to $\infty$ for small $\gamma$. The number $\alpha_{x}\left(\left\{x_{i}\right\}, L\right)$ marks the transition point between these two behaviours.

By Remark 2.2(b) if $\left\{x_{i}^{\prime}\right\}$ is a subsequence of $\left\{x_{i}\right\}$ then $\alpha_{x}\left(\left\{x_{i}^{\prime}\right\}, L\right) \leqslant \alpha_{x}\left(\left\{x_{i}\right\}, L\right)$. Thus we may freely replace a sequence with a subsequence when trying to establish lower bounds.

Definition 2.4 Let $k$ be a number field, $X$ a projective variety over $\operatorname{Spec}(k), L$ a line bundle on $X$, and $x \in X(\bar{k})$. Then $\alpha_{x}(L)$ is defined to be the infimum of all approximation constants of sequences of points in $X(k)$ converging to $x$. If no such sequence exists then set $\alpha_{x}(L)=\infty$.

To see the connection with the usual approximation exponent on $\mathbb{P}^{1}$, suppose that $L$ is an ample line bundle. We may define an approximation constant $\tau_{x}(L)$ by simply extending the definition on $\mathbb{P}^{1}$, namely by defining $\tau_{x}(L)$ to be the unique extended real number $\tau_{x}(L) \in[0, \infty]$ such that the inequality

$$
d_{v}(x, y)<\frac{1}{H_{L}(y)^{\tau_{x}(L)+\delta}}
$$

has only finitely many solutions $y \in X(k)$ whenever $\delta>0$ (respectively has infinitely many solutions $y \in X(k)$ whenever $\delta<0)$. Then [10, Proposition 2.11] implies that $\alpha_{x}(L)=1 / \tau_{x}(L)$. In particular the theorem of Liouville becomes $\alpha_{x}\left(\mathcal{O}_{\mathbb{P}^{1}}(1)\right) \geqslant 1 / d$ for $x \in \mathbb{R}$ of degree $d$ over $\mathbb{Q}$, and it is this type of lower bound that we wish to generalize to arbitrary varieties. The choice of using the reciprocal of $\tau$ is justified by the resulting formal similarity with the Seshadri constant, and more natural behaviour when we vary $L$ (see, for example, Proposition 2.11).

We need two results on $\alpha_{x}$ before continuing onto the Seshadri constant. First, we will need to know how to calculate $\alpha_{x}$ in one simple case. 
Lemma 2.5 ([10, Lemma 2.13]) Let $x \in \mathbb{P}^{n}(k)$. Then $\alpha_{x, \mathbb{P}^{n}}\left(\mathcal{O}_{\mathbb{P}^{n}}(1)\right)=1$.

Second, it will be useful to know how the approximation constant changes when we change the field $k$. Let $K / k$ be a finite extension with $K \subset \bar{k}$, and set $X_{K}=X \times{ }_{k} K$. A point $y \in X(\bar{k})$ is a map $\operatorname{Spec}(\bar{k}) \rightarrow X$ over $\operatorname{Spec}(k)$, and factors through the map $X_{K} \rightarrow X$, i.e., such a point $x$ gives a point of $X_{K}(\bar{k})$. We thus have a canonical identification $X_{K}(\bar{k})=X(\bar{k})$ which we use implicitly in the paragraphs below. We use the notation that $\alpha_{x}\left(\left\{x_{i}\right\}, L\right)_{K}$ (respectively $\left.\alpha_{x}(L)_{K}\right)$ denotes the approximation constant of a sequence (respectively point $x$ ) computed on $X_{K}$ with respect to $K$. This means that when computing $\alpha$, we use the height $H_{L}$ relative to $K$ and normalize $d_{v}$ relative to $K$ (we define $d_{v}$ on $X_{K}$ using the same embedding used to define $d_{v}$ on $X$ ). If $d=[K: k]$ and $m_{v}=\left[K_{v}: k_{v}\right]$ (where $K_{v}$ and $k_{v}$ denote the completions of $K$ and $k$ with respect to $v$ ) then this means simply that $H_{L}\left(x_{i}\right)_{K}=H_{L}\left(x_{i}\right)_{k}^{d}$ and $d_{v}\left(x, x_{i}\right)_{K}=d_{v}\left(x, x_{i}\right)_{k}^{m_{v}}$.

Proposition 2.6 Let $X$ be a variety over $\operatorname{Spec}(k), x \in X(\bar{k})$ any point, L a line bundle on $X$, and $\left\{x_{i}\right\} \rightarrow x$ a sequence of points in $X(k)$ approximating $x$. Let $K$ be any finite extension of $k$ and set $X_{K}=X \times_{k} K$. The base change of $\left\{x_{i}\right\}$ gives a sequence $\left\{y_{i}\right\}$ of $K$-points of $X_{K}$ approximating $x$. Set $m_{v}=\left[K_{v}: k_{v}\right]$, and let $d=[K: k]$. Then

$$
\alpha_{x}\left(\left\{y_{i}\right\}, L\right)_{K}=\frac{d}{m_{v}} \alpha_{x}\left(\left\{x_{i}\right\}, L\right)_{k} .
$$

In particular, we have the bound $\alpha_{x}(L)_{K} \leqslant d \alpha_{x}(L)_{k} / m_{v}$.

Proof The claim that $\alpha_{x}\left(\left\{x_{i}\right\}, L\right)_{K}=d \alpha_{x}\left(\left\{x_{i}\right\}, L\right)_{k} / m_{v}$ follows immediately from the equalities $H_{L}(\cdot)_{K}=H_{L}(\cdot)_{k}^{d}$ and $d_{v}(\cdot, \cdot)_{K}=d_{v}(\cdot, \cdot)_{k}^{m_{v}}$. The inequality $\alpha_{x}(L)_{K} \leqslant d \alpha_{x}(L)_{k} / m_{v}$ then follows since the sequences in $X_{K}(K)$ approximating $x$ which come from sequences $\left\{x_{i}\right\}$ in $X(k)$ are a subset of all the sequences in $X_{K}(K)$ approximating $x$.

Remark 2.7 Let $x$ be a point of $X(\bar{k})$ and let $K$ be the field of definition of $x$. If $K \nsubseteq k_{v}$, or equivalently, $K_{v} \neq k_{v}$ then it will be impossible to find a sequence of points of $X(k)$ converging (in terms of $d_{v}$ ) to $x$. For example, when $v$ is archimedean this happens when $k_{v}=\mathbb{R}$ and $K_{v}=\mathbb{C}$. Thus, if we can approximate $x$ by points of $X(k)$ we may assume that $K_{v}=k_{v}$ and so $m_{v}=1$.

The following result (appearing in [10] as Theorem 2.16 and in [9] as Theorem 2.8, although the latter version is incorrectly stated) is obtained by combining Roth's and Dirichlet's Theorems for approximation on $\mathbb{P}^{1}$, as well as the local information about the singularity type. It shows how to calculate $\alpha_{x}$ on any singular $k$-rational curve.

Theorem 2.8 Let $C$ be any singular $k$-rational curve and $\varphi: \mathbb{P}^{1} \rightarrow C$ the normalization map. Then for any ample line bundle $L$ on $C$, and any $x \in C(\bar{k})$ we have the equality

$$
\alpha_{x, C}(L)=\min _{q \in \varphi^{-1}(x)} \frac{d}{r_{q} m_{q}}
$$


where $d=\operatorname{deg}(L), m_{q}$ is the multiplicity of the branch of $C$ through $x$ corresponding to $q$, and

$$
r_{q}= \begin{cases}0 & \text { if } \kappa(q) \nsubseteq k_{v} \\ 1 & \text { if } \kappa(q)=k \\ 2 & \text { otherwise. }\end{cases}
$$

Here $\kappa(q)$ means the residue field of the point $q$, and we use $r_{q}=0$ as a shorthand for $d / r_{q} m_{q}=\infty$.

The Seshadri constant. The Seshadri constant was introduced by Demailly in [4] for the purposes of measuring the local positivity of a line bundle.

Definition 2.9 Let $X$ be a projective variety, $x$ a point of $X$, and $L$ a nef line bundle on $X$. The Seshadri constant, $\epsilon_{x}(L)$, is defined to be

$$
\epsilon_{x}(L)=\sup \left\{\gamma \geqslant 0: \pi^{*} L-\gamma E \text { is nef }\right\}
$$

where $\pi: \widetilde{X} \rightarrow X$ is the blowup of $X$ at $x$, with exceptional divisor $E$.

A basic property of the Seshadri constant is that if $L$ is ample (as opposed to just being nef) then $\pi^{*} L-\gamma E$ is itself ample on $\widetilde{X}$ for all rational $\gamma \in\left(0, \epsilon_{x}(L)\right)$. An argument for this appears in the original paper of Demailly [4] defining the Seshadri constant ${ }^{1}$.

In the discussion of Conjecture 4.2 below we will need the following alternate characterization of the Seshadri constant:

Proposition 2.10 ([7, Proposition 5.1.5]) With the same setup as in Definition 2.9,

$$
\epsilon_{x}(L)=\inf _{x \in C \subseteq X}\left\{\frac{L \cdot C}{\operatorname{mult}_{x}(C)}\right\}
$$

where the infimum is taken over all reduced irreducible curves $C$ passing through $x$.

In order to indicate the parallels between $\alpha_{x}$ and $\epsilon_{x}$, and for use below, we list a few of their formal properties here.

Proposition 2.11 Let $X$ be a projective variety over $\operatorname{Spec}(k), x \in X(\bar{k})$, and let $L$ be any nef line bundle on $X$.

(a) For any positive integer $m, \alpha_{x}(m L)=m \alpha_{x}(L)$ and $\epsilon_{x}(m L)=m \epsilon_{x}(L)$. (Thus $\alpha$ and $\epsilon$ also make sense for nef $\mathbb{Q}$-divisors.)

(b) $\alpha_{x}$ and $\epsilon_{x}$ are concave functions of L: for any positive rational numbers $a$ and $b$, and any $\mathbb{Q}$-divisors $L_{1}$ and $L_{2}$ (again defined over $k$, but with the exception of the case that $\left.\left\{\alpha_{x}\left(L_{1}\right), \alpha_{x}\left(L_{2}\right)\right\}=\{-\infty, \infty\}\right)$ we have

$$
\alpha_{x}\left(a L_{1}+b L_{2}\right) \geqslant a \alpha_{x}\left(L_{1}\right)+b \alpha_{x}\left(L_{2}\right)
$$

\footnotetext{
$\overline{1 \text { This property appears as the statement " } F_{p, q} \text { is ample whenever } p}>q / \epsilon_{x}(L)$ " in [4, p. 98].
} 
and

$$
\epsilon_{x}\left(a L_{1}+b L_{2}\right) \geqslant a \epsilon_{x}\left(L_{1}\right)+b \epsilon_{x}\left(L_{2}\right)
$$

where for the last inequality we assume that $L_{1}$ and $L_{2}$ are nef.

(c) If $Z$ is a subvariety of $X$ over $\operatorname{Spec}(k)$ then for any point $z \in Z(\bar{k})$ we have $\alpha_{z}\left(\left.L\right|_{Z}\right) \geqslant \alpha_{z}(L)$ and $\epsilon_{z}\left(\left.L\right|_{Z}\right) \geqslant \epsilon_{z}(L)$.

(d) If $Y$ is also a variety over $\operatorname{Spec}(k), x \in X(k), y \in Y(k)$ and $L_{X}$ and $L_{Y}$ are nef line bundles on $X$ and $Y$ respectively then

$$
\alpha_{x \times y, X \times Y}\left(L_{X} \boxplus L_{Y}\right)=\min \left(\alpha_{x, X}\left(L_{X}\right), \alpha_{y, Y}\left(L_{Y}\right)\right)
$$

and

$$
\epsilon_{x \times y, X \times Y}\left(L_{X} \boxplus L_{Y}\right)=\min \left(\epsilon_{x, X}\left(L_{X}\right), \epsilon_{y, Y}\left(L_{Y}\right)\right) .
$$

Note that by $L_{X} \boxplus L_{Y}$ we mean the line bundle $\operatorname{pr}_{X}^{*} L_{1}+\operatorname{pr}_{Y}^{*} L_{2}$ on $X \times Y$, where $\operatorname{pr}_{X}$ and $\operatorname{pr}_{Y}$ are the projections. We prefer additive notation for line bundles since this is in line with the behaviour of $\alpha_{x}$ and $\epsilon_{x}$, and hence use $L_{X} \boxplus L_{Y}$ rather than $L_{1} \otimes L_{2}$.

Proof All the proofs follow from elementary arguments using the definitions. For the statements about $\alpha_{x}$ see [10, Proposition 2.14], and for the statements about $\epsilon_{x}$ see [10, Proposition 3.4].

\section{A Liouville lower bound for $\alpha$}

In this section, as in the previous one, we fix a number field $k$ and let $X$ be a projective variety over $\operatorname{Spec}(k)$.

Lemma 3.1 Let $x$ be a point of $X(k)$, and $\pi: \widetilde{X} \rightarrow X$ the blowup of $X$ at $x$ with exceptional divisor $E$. Choose an embedding $\varphi: X \hookrightarrow \mathbb{P}^{n}$ so that $x \mapsto[1: 0: \cdots: 0]$. Let $Z_{0}, \ldots, Z_{n}$ be the coordinates on $\mathbb{P}^{n}$ and define functions $u_{i}, i=1, \ldots, n$, on the open subset where $Z_{0} \neq 0$ by $u_{i}=Z_{i} / Z_{0}$. For each place $v$ of $k$, define a function $e_{v}: X\left(k_{v}\right) \rightarrow \mathbb{R}_{\geqslant 0}$ by

$$
e_{v}(y)= \begin{cases}1 & \text { if } Z_{0}(y)=0, \\ \min \left(1, \max \left(\left\|u_{1}(y)\right\|_{v}, \ldots,\left\|u_{n}(y)\right\|_{v}\right)\right) & \text { if } Z_{0}(y) \neq 0 .\end{cases}
$$

Then

(a) $0 \leqslant e_{v} \leqslant 1$ for all places $v$.

(b) $e_{v}(\cdot)$ is equivalent to $d_{v}(x, \cdot)$ as a function on $X\left(k_{v}\right)$.

(c) For $y \in X(k), y \neq x$, we have $H_{E}(y)=\left(\prod_{w} e_{w}(y)\right)^{-1}$.

Proof Part (a) is clear from the definition. We now prove (b). By [10, Proposition 2.4], distance functions associated to different projective embeddings of $X$ are equivalent on 
$X\left(k_{v}\right) \times X\left(k_{v}\right)$, so we may assume that $d_{v}$ has been defined using the embedding $\varphi$. If $v$ is non-archimedean, then the formula for the distance function in the non-archimedean case and the fact that $x$ is sent to $[1: 0: \cdots: 0]$ give

$$
d_{v}(x, y)=\frac{\max \left(\left\|Z_{1}(y)\right\|_{v},\left\|Z_{2}(y)\right\|_{v}, \ldots,\left\|Z_{r}(y)\right\|_{v}\right)}{\max \left(\left\|Z_{0}(y)\right\|_{v},\left\|Z_{1}(y)\right\|_{v}, \ldots,\left\|Z_{r}(y)\right\|_{v}\right)} \quad \text { for all } \quad y \in X(\bar{k}) \text {. }
$$

For $y \in X\left(k_{v}\right)$, this is equal to $e_{v}(y)$.

In the case that $v$ is archimedean, we may further assume that $k_{v}=\mathbb{C}$, since the functions to be compared transform the same way under field extensions. From the formula for the distance in the archimedean case and the fact that $x$ is sent to $[1: 0: \cdots: 0]$ we obtain

$$
\begin{aligned}
d_{v}(x, y)=1-\frac{\left|y_{0}\right|^{2}}{\left|y_{0}\right|^{2}+\cdots+\left|y_{r}\right|^{2}} & =\frac{\left|y_{1}\right|^{2}+\cdots+\left|y_{r}\right|^{2}}{\left|y_{0}\right|^{2}+\left|y_{1}\right|^{2}+\cdots+\left|y_{r}\right|^{2}} \\
& =\frac{\left\|y_{1}\right\|_{v}+\cdots+\left\|y_{r}\right\|_{v}}{\left\|y_{0}\right\|_{v}+\left\|y_{1}\right\|_{v}+\cdots+\left\|y_{r}\right\|_{v}} .
\end{aligned}
$$

For $y \in U\left(k_{v}\right), y_{0} \neq 0$, and $u_{j}(y)=y_{j} / y_{0}$ for $j=1, \ldots, r$. Thus $d_{v}(x, y)=$ $\left(\left\|u_{1}(y)\right\|_{v}+\cdots+\left\|u_{r}(y)\right\|_{v}\right) /\left(1+\left\|u_{1}(y)\right\|_{v}+\cdots+\left\|u_{r}(y)\right\|_{v}\right)$; it is then elementary to check that

$$
\frac{1}{r} d_{v}(x, y) \leqslant \max \left(\left\|u_{1}(y)\right\|_{v}, \ldots,\left\|u_{n}(y)\right\|_{v}\right) \leqslant 2 d_{v}(x, y),
$$

for all $y \in U\left(k_{v}\right)$. For $y \in X\left(k_{v}\right) \backslash U\left(k_{v}\right)$ we have $1=d_{v}(x, y)=e_{v}(y)$, and thus $d_{v}(x, \cdot)$ is equivalent to $e_{v}(\cdot)$ on $X\left(k_{v}\right)$.

In (c) we are considering points $y \in X(k), y \neq x$ also to be points of $\widetilde{X}(k)$ via the birational map $\pi$. To prove (c) it suffices, by using the functoriality of heights under pullback, to consider the case that $X=\mathbb{P}^{n}$. Then the blowup $\widetilde{\mathbb{P}}^{n}$ of $\mathbb{P}^{n}$ at $x$ is a subvariety of $\mathbb{P}^{n} \times \mathbb{P}^{n-1}$ and $\mathcal{O}_{\widetilde{\mathbb{P}}^{n}}(E)$ is the restriction of $\mathcal{O}_{\mathbb{P}^{n} \times \mathbb{P}^{n-1}}(1,-1)$ to $\widetilde{\mathbb{P}}^{n}$. From this description of $\mathcal{O}_{\widetilde{\mathbb{P}}^{n}}(E)$ we obtain the formula

$$
H_{E}(y)=\prod_{w} \frac{\max \left(\left\|Z_{0}(y)\right\|_{w},\left\|Z_{1}(y)\right\|_{w}, \ldots,\left\|Z_{n}(y)\right\|_{w}\right)}{\max \left(\left\|Z_{1}(y)\right\|_{w}, \ldots,\left\|Z_{n}(y)\right\|_{w}\right)}
$$

from which (c) follows easily.

For the following lemma, we will need an additional definition. The stable base locus of a line bundle $L$ is the intersection of the base loci of $m L$ as $m \rightarrow \infty$. For details, see [7, Definition 2.1.20].

Lemma 3.2 Suppose that $x \in X(k)$ and let $\pi: \widetilde{X} \rightarrow X$ be the blowup at $x$ with exceptional divisor $E$. Let $L$ be a line bundle on $X$ and $\gamma>0$ a rational number such that $L_{\gamma}=\pi^{*} L-\gamma E$ is in the effective cone of $\widetilde{X}$. Let $B^{\prime}$ be the stable base locus of $L_{\gamma}$ and set $B=\pi\left(B^{\prime}\right)$. 
Then there is a positive real constant $M$ (depending only on $x$ and $L$ ) such that for any sequence of $k$-points $\left\{x_{i}\right\} \rightarrow x$ with all points of $\left\{x_{i}\right\}$ outside of $B$, we have

$$
H_{L}\left(x_{i}\right) d_{v}\left(x, x_{i}\right)^{\gamma} \geqslant M \text {. }
$$

In particular, $\alpha\left(\left\{x_{i}\right\}, L\right) \geqslant \gamma$.

Proof Let $U=\widetilde{X} \backslash B^{\prime}$. Since $B^{\prime}$ is the stable base locus of $L_{\gamma}$ there is a constant $c$ (depending only on $x$ and $L$ ) so that $H_{L_{\gamma}}(y) \geqslant c$ for all $y \in U(k)$. Applying Lemma 3.1, we then have

$$
\begin{aligned}
c \leqslant H_{L_{\gamma}}\left(x_{i}\right) & =H_{L}\left(x_{i}\right) H_{E}\left(x_{i}\right)^{-\gamma} \\
\stackrel{\text { Lemma 3.1 (c) }}{=} & H_{L}\left(x_{i}\right)\left(\prod_{w} e_{w}\left(x_{i}\right)\right)^{\gamma} \stackrel{\text { Lemma 3.1 (a) }}{\leqslant} H_{L}\left(x_{i}\right) e_{v}\left(x_{i}\right)^{\gamma} .
\end{aligned}
$$

By Lemma 3.1 (b), $d_{v}\left(x, x_{i}\right)$ and $e_{v}\left(x_{i}\right)$ are equivalent functions on $X(k)$ and therefore $H_{L}\left(x_{i}\right) d_{v}\left(x, x_{i}\right)^{\gamma} \geqslant M$ for some positive constant $M$, again depending only on $x$ and $L$.

For any $\delta>0$ we thus have $H_{L}\left(x_{i}\right) d_{v}\left(x, x_{i}\right)^{\gamma-\delta} \geqslant M d_{v}\left(x, x_{i}\right)^{-\delta}$ and so conclude that $\gamma-\delta \notin A\left(\left\{x_{i}\right\}, L\right)$ since $c^{\prime} d_{v}\left(x, x_{i}\right)^{-\delta} \rightarrow \infty$ as $i \rightarrow \infty$. Therefore $\gamma \leqslant$ $\alpha\left(\left\{x_{i}\right\}, L\right)$.

The main result of this section is the following implication of Lemma 3.2.

Theorem 3.3 (Liouville-type Theorem) Let $X$ be an algebraic variety over $\operatorname{Spec}(k)$, $x \in X(\bar{k})$ any point, and set $d=[K: k]$ where $K$ is the field of definition of $x$. Set $X_{K}=X \times_{k} K$, let $\widetilde{X}$ be the blowup of $X_{K}$ at $x$ with exceptional divisor $E$, and set $\pi$ to be the composite $\pi: \widetilde{X} \rightarrow X_{K} \rightarrow X$. Let L be a nef line bundle on $X$, and $\gamma>0$ a rational number such that $L_{\gamma}=\pi^{*} L-\gamma E$ is in the effective cone of $\widetilde{X}$. Finally let $B^{\prime}$ be the stable base locus of $L_{\gamma}$ and set $B=\pi\left(B^{\prime}\right)$. Then there is a positive real constant $M$ such that for all $y \in X(k) \backslash B(k)$, we have $H_{L}(y) d_{v}(x, y)^{\gamma / d} \geqslant M$, and

(a) For any sequence $\left\{x_{i}\right\} \rightarrow x$ of $k$-points approximating $x$, if infinitely many points of $\left\{x_{i}\right\}$ are outside $B$ then $\alpha\left(\left\{x_{i}\right\}, L\right) \geqslant \gamma / d$.

(b) If $\alpha_{x}(L)<\gamma / d$ then $x \in B$ and $\alpha_{x}(L)=\alpha_{x}\left(\left.L\right|_{B}\right)$.

(c) If $x \in B$ and $\alpha_{x}\left(\left.L\right|_{B}\right) \geqslant \gamma / d$ then $\alpha_{x}(L) \geqslant \gamma / d$.

Furthermore, there is a subvariety $Y$ of $X$ such that $x \in Y$ and for all $y \in X(k)$, we have $H_{L}(y) d_{v}(x, y)^{\gamma / d} \geqslant M$, provided that $\left.(L-\gamma E)\right|_{Y}$ is in the effective cone of $Y$.

Proof Let $\left\{x_{i}\right\}$ be a sequence approximating $x$. If infinitely many $x_{i}$ lie outside of $B$ then we may pass to the subsequence of points outside of $B$, which can only approximate the point $x$ better than the sequence as a whole. To prove part (a) we may therefore assume that all points of $\left\{x_{i}\right\}$ lie outside $B$. Base changing $\left\{x_{i}\right\}$ we obtain a sequence $\left\{y_{i}\right\}$ in $X_{K}(K)$ approximating $x \in X_{K}(K)$. Applying Lemma 3.2 to $X_{K}$, we conclude that $H_{L}\left(y_{i}\right) d_{v}\left(x, y_{i}\right)^{\gamma} \geqslant M$ and $\alpha\left(\left\{y_{i}\right\}, L\right)_{K} \geqslant \gamma$. Since there is a sequence of $k$-points approximating $x$ we conclude by Remark 2.7 that (in the notation 
of Proposition 2.6) $m_{v}=1$. Therefore by Proposition 2.6, $H_{L}\left(y_{i}\right) d_{v}\left(x, y_{i}\right)^{\gamma / d} \geqslant M$ and $\alpha\left(\left\{x_{i}\right\}, L\right)_{k}=\alpha\left(\left\{y_{i}\right\}, L\right)_{K} / d \geqslant \gamma / d$, proving (a).

If $\alpha_{x}(L)<\gamma / d$ then there must be a sequence $\left\{x_{i}\right\}$ approximating $x$ such that $\alpha\left(\left\{x_{i}\right\}, L\right)<\gamma / d$. By part (a), this implies that all but finitely many $x_{i}$ lie in $B$. Thus $x \in B$ since $B$ is closed. Since omitting finitely many elements of a sequence does not change the approximation constant we may assume that all $x_{i}$ are contained in $B$. Since $\alpha_{x, X}(L)$ is the infimum of the approximation constants for sequences $\left\{x_{i}\right\}$ with $\alpha\left(\left\{x_{i}\right\}, L\right)<\gamma / d$ we conclude that $\alpha_{x}(L)=\alpha_{x}\left(\left.L\right|_{B}\right)$ proving $(\mathrm{b})$.

If $\alpha_{x}(L)<\gamma / d$ then part (b) along with the hypothesis for part (c) lead to an immediate contradiction. Thus, under the hypotheses of part (c), $\alpha_{x}(L) \geqslant \gamma / d$.

The final remark is obvious in cases (a) and (c), and in case (b), we may replace $X$ with $B$ and $L$ with $\left.L\right|_{B}$ and apply Theorem 3.3 again. Iterating this, we deduce the desired result.

Remark 3.4 Theorem 3.3 still holds if we replace $B$ by the Zariski closure of $B(k)$. This has the added advantage that every component of $B$ is then absolutely irreducible (see [10, Lemma 2.17]).

Corollary 3.5 Suppose that $L$ is a nef line bundle on $X$ such that $\pi^{*} L-\gamma E$ has empty stable base locus for all rational $\gamma \in\left(0, \epsilon_{x}(L)\right)$. Then $\alpha_{x}(L) \geqslant \epsilon_{x}(L) / d$.

Proof For all rational $\gamma \in\left(0, \epsilon_{x}(L)\right)$ the stable base locus of $\pi^{*} L-\gamma E$ is empty by hypothesis, and thus, by Theorem 3.3 (a), we conclude that $\alpha_{x}(L) \geqslant \gamma / d$ for any such $\gamma$, and hence that $\alpha_{x}(L) \geqslant \epsilon_{x}(L) / d$.

Corollary 3.6 If $L$ is an ample line bundle, then $\alpha_{x}(L) \geqslant \epsilon_{x}(L) / d$.

Proof For all rational $\gamma \in\left(0, \epsilon_{x}(L)\right)$ the line bundle $\pi^{*} L-\gamma E$ is ample on $\widetilde{X}$. (See the discussion after Definition 2.9 for a proof of this.) In particular, the stable base locus of $\pi^{*} L-\gamma E$ is empty, so the result follows by Corollary 3.5.

Remark 3.7 If $X=\mathbb{P}^{1}$ then Corollary 3.6 and the fact that $\epsilon_{x}\left(\mathcal{O}_{\mathbb{P}^{n}}(1)\right)=1$ give $\alpha_{x}\left(\mathcal{O}_{\mathbb{P}^{1}}(1)\right) \geqslant 1 / d$. Thus on $\mathbb{P}^{1}$ Corollary 3.6 amounts to the classic Liouville bound $\tau_{x} \leqslant d$. Liouville's original result also includes an explicit relation between the height and distance of approximating rational points, and Theorem 3.3 has this feature as well. For this reason we consider Theorem 3.3 and its corollaries to be "Liouville bounds" for $\alpha_{x}$.

The effective cone is usually larger than the nef cone, and in general the parts of Theorem 3.3 imply a much stronger lower bound for $\alpha_{x}(L)$ than Corollary 3.5. We will use this in the next section to compute $\alpha$ for the cubic surface, but give a brief illustration now by calculating $\alpha$ for rational points of a non-split quadric surface in $\mathbb{P}^{3}$. (A split quadric surface is isomorphic to $\mathbb{P}^{1} \times \mathbb{P}^{1}$, and $\alpha_{x}\left(\mathcal{O}_{\mathbb{P}^{1} \times \mathbb{P}^{1}}(a, b)\right)=\min (a, b)$ when $a, b>0$, as implied by Proposition 2.11 (d) and computed in both [9, Theorem 3.1] and [10, Section 2; Example (b) just before Lemma 2.17].)

Example 3.8 Let $X$ be a smooth quadric surface in $\mathbb{P}^{3}$ defined over $k$, and set $L=$ $\left.\mathcal{O}_{\mathbb{P}^{3}}(1)\right|_{X}$. We assume that no lines on $X$ are defined over $k$. Let $x$ be a $k$-point 
of $X$. By intersecting with a (rationally defined) hyperplane we may find a conic $C$ passing through $x$ such that $C$ is isomorphic to $\mathbb{P}^{1}$ over $k$. By Lemma 2.5 and Proposition $2.11(\mathrm{a}, \mathrm{c})$, we therefore have $\alpha_{x, X}(L) \leqslant \alpha_{x, C}\left(\left.L\right|_{C}\right)=\alpha_{x, \mathbb{P}^{1}}\left(\mathcal{O}_{\mathbb{P}^{1}}(2)\right)=$ 2. Since $x$ lies on a line (over $\bar{k}$ ), we have $\epsilon_{x}(L)=1$, and, applying Corollary 3.5, we obtain $\alpha_{x}(L) \geqslant 1$. Thus $1 \leqslant \alpha_{x}(L) \leqslant 2$, i.e., Corollary 3.5 does not give enough information to determine $\alpha_{X}(L)$ in this case.

However, let $\pi: \widetilde{X} \rightarrow X$ be the blowup of $X$ at $x$ with exceptional divisor $E$. Then $\pi^{*} L-2 E$ is effective with base locus the proper transform of the two lines passing through $x$. In particular the image $B$ of this base locus is the union of the two lines of ruling passing through $x$. Since (by assumption) neither of these lines is defined over $k, x$ is the only $k$-point of $B$. Thus, by Theorem 3.3 (a), if $\left\{x_{i}\right\}$ is any sequence of $k$-points approximating $x$ then $\alpha\left(\left\{x_{i}\right\}, L\right) \geqslant 2$, and in particular $\alpha_{x}(L) \geqslant 2$. Thus $\alpha_{x}(L)=2$ for all $k$-points of $X$.

Since $X$ is non-split the Picard group of $X$ (over $k$ ) has rank one with generator $L$. Thus the above computation and the homogeneity in Proposition 2.11 (a) determines $\alpha$ for all $x \in X(k)$ and all ample line bundles on $X$ defined over $k$.

\section{The cubic surface}

In this section, we will compute $\alpha_{x}$ and $\epsilon_{x}$ for all $k$-rational points $x$ on the blowup $X$ of $\mathbb{P}^{2}$ at six $k$-rational points in general position. To begin, we will recall some notions from [9].

Definition 4.1 A sequence $\left\{x_{i}\right\} \rightarrow x$ whose approximation constant is equal to $\alpha_{x}(L)$ (if such a sequence exists) is called a sequence of best approximation to $x$. A curve $C$ passing through $x$ is a called a curve of best approximation (with respect to $L$ ) if $C$ contains a sequence of best approximation to $x$.

In other words, if $C$ is a curve of best approximation to $x$ on $X$, then the rational points on $C$ approximate $x$ roughly as well as the rational points on $X$ approximate $x$.

Note also that for a point $x$ on a curve $C$ and anple divisor $L$ on $C$, there is always a sequence $\left\{x_{i}\right\}$ such that $\alpha_{x}\left(\left\{x_{i}\right\}, L\right)=\alpha_{x}(L)$. Thus, in particular, $C$ is a curve of best approximation if and only if $\alpha_{x}\left(\left.L\right|_{C}\right)=\alpha_{x}(L)$.

In the example of the non-split quadric — and in many others considered in [9]— there is always a curve of best approximation to $x$. In [9, Section 4], it is shown that if Vojta's main conjectures (see [13] for statements) are true, then $\alpha_{x}(L)$ finite implies that $\alpha_{x}(L)$ is computed on a subvariety $V \subseteq X$ of negative Kodaira dimension (possibly $X$ itself, if $X$ has negative Kodaira dimension). Since varieties of negative Kodaira dimension are (again, conjecturally) covered by rational curves, one is led to the following further prediction:

Conjecture 4.2 ([9, Conjecture 2.7]) Let $X$ be an algebraic variety defined over $k$, and $L$ any ample divisor on $X$. Let $x$ be any $k$-rational point on $X$ and assume that there is a rational curve defined over $k$ passing through $x$. Then there exists a curve $C$ (necessarily rational) of best approximation to $x$ on $X$ with respect to $L$. 
In [9], the first author proves this conjecture in many cases, and shows that in many others it follows from Vojta's Conjecture. Those proofs use a slightly different definition of $\alpha$, but the proofs do not essentially change in the new setting.

The Seshadri-constant analogue of a curve of best approximation is called a Seshadri curve (cf. Proposition 2.10):

Definition 4.3 Let $L$ be a nef divisor on an algebraic variety $X$, and $x \in X$ any point. A Seshadri curve for $x$ with respect to $L$ is a curve $C$ such that $\epsilon_{x, X}(L)=$ $(L \cdot C) / \operatorname{mult}_{x}(C)$.

In all currently known examples, there exists a Seshadri curve for $x$ with respect to $L$, but it is conjectured that this is not always the case. In particular, it is possible that the Seshadri constant might sometimes be irrational (see [7, Remark 5.1.13]).

It is useful to know that for a fixed curve $C$, the set of line bundles for which $C$ is a curve of best approximation form a subcone of the Néron-Severi group, and similarly for the property of being a Seshadri curve.

Proposition 4.4 Let $X$ be a variety over $\operatorname{Spec}(k)$, and let $x \in X(k)$ be any $k$-rational point. Let $D_{1}$ and $D_{2}$ be nef divisors on $X$ with height functions $H_{1}$ and $H_{2}$ bounded below by a positive constant in some neighbourhood of $x$. Let $a_{1}$ and $a_{2}$ be non-negative integers, and let $D=a_{1} D_{1}+a_{2} D_{2}$.

(a) If $C$ is a curve of best approximation for $D_{1}$ and $D_{2}$, then $C$ is also a curve of best approximation for $D$.

(b) If $C$ is a Seshadri curve for $x$ with respect to $D_{1}$ and $D_{2}$, then $C$ is also a Seshadri curve for $x$ with respect to $D$.

Proof Part (a) appears as [9, Corollary 3.2]. To prove part (b), note that Proposition 2.11 (b) implies the estimate

$$
\epsilon_{x}\left(a_{1} D_{1}+a_{2} D_{2}\right) \geqslant a_{1} \epsilon_{x}\left(D_{1}\right)+a_{2} \epsilon_{x}\left(D_{2}\right) .
$$

On the other hand, the hypotheses of part (b) give

$$
\begin{aligned}
\frac{C \cdot D}{\operatorname{mult}_{x}(C)}=\frac{C \cdot\left(a_{1} D_{1}+a_{2} D_{2}\right)}{\operatorname{mult}_{x} C} & =\frac{a_{1}\left(C \cdot D_{1}\right)}{\operatorname{mult}_{x}(C)}+\frac{a_{2}\left(C \cdot D_{2}\right)}{\operatorname{mult}_{x}(C)} \\
& =a_{1} \epsilon_{x}\left(D_{1}\right)+a_{2} \epsilon_{x}\left(D_{2}\right) .
\end{aligned}
$$

Thus, by Proposition 2.10, $a_{1} \epsilon_{x}\left(D_{1}\right)+a_{2} \epsilon_{x}\left(D_{2}\right)$ is an upper bound for $\epsilon_{x}(D)$. Therefore $\epsilon_{x}\left(a_{1} D_{1}+a_{2} D_{2}\right)=a_{1} \epsilon_{x}\left(D_{1}\right)+a_{2} \epsilon_{x}\left(D_{2}\right)$ and $C$ is a Seshadri curve for $D$, proving (b).

We are now ready to begin the proof of the main result of this section. Before we state and prove the general result, we will illustrate the fundamental techniques in the case $L=-K$.

Theorem 4.5 Let $X$ be a smooth cubic surface in $\mathbb{P}^{3}$ defined over $k$, and isomorphic over $k$ to the blowup of $\mathbb{P}^{2}$ at six $k$-rational points in general position. Let $x \in X(k)$ be any $k$-rational point, and let $C_{x}$ be the curve of intersection of $X$ with the tangent plane to $X$ at $x$. Then 


$$
\epsilon_{x}(-K)= \begin{cases}1 & \text { if } x \text { lies on one of the } 27 \text { lines of } X \\ \frac{3}{2} & \text { otherwise }\end{cases}
$$

while

$$
\begin{aligned}
& \alpha_{x}(-K)= \begin{cases}\frac{1}{3} & \begin{array}{l}
\text { if } x \text { lies on one of the } 27 \text { lines of } X ; \\
\frac{1}{2}
\end{array} \\
& \circ C_{x} \text { is cuspidal at } x, \text { or } \\
& \circ C_{x} \text { is nodal at xwith tangent lines having } \\
& \text { slopes in } k_{v} \text { but not } k ; \\
2 & \text { otherwise. }\end{cases} \\
& \text { (i.e., } C_{x} \text { is nodal at } x \text {, and the slopes of } \\
& \text { the tangent lines are in } k \text { or not in } k_{v} \text {.) }
\end{aligned}
$$

Proof Set $L=-K=\left.\mathcal{O}_{\mathbb{P}^{3}}(1)\right|_{X}$, and let $x$ be a point of $X(k)$. If $x$ lies on a line $\ell$ then, by Proposition 2.11 (c), we have $\epsilon_{x, \ell}\left(\left.L\right|_{\ell}\right) \geqslant \epsilon_{x, X}(L) \geqslant \epsilon_{x, \mathbb{P}^{3}}\left(\mathcal{O}_{\mathbb{P}^{3}}(1)\right)$. Since $\epsilon_{x, \ell}\left(\left.L\right|_{\ell}\right)=\epsilon_{x, \mathbb{P}^{3}}\left(\mathcal{O}_{\mathbb{P}^{3}}(1)\right)=1$, we conclude that $\epsilon_{x}(L)=1$. Similarly (using Proposition 2.11 (c) again and the fact that $\alpha_{x, \ell}\left(\left.L\right|_{\ell}\right)=\alpha_{x, \mathbb{P}^{3}}\left(\mathcal{O}_{\mathbb{P}^{3}}(1)\right)=1$ by Lemma 2.5) we conclude that $\alpha_{x}(L)=1$.

We now suppose that $x$ does not lie on a line. Let $\pi: \widetilde{X} \rightarrow X$ be the blowup of $X$ at $x$, with exceptional divisor $E$. Then $C_{X}$ is a Seshadri curve for $x$ with respect to $L$. To see this, note first that $C_{x}$ satisfies $C_{x} \cdot L /$ mult $_{x}\left(C_{x}\right)=3 / 2$, so $\epsilon_{x}(L) \leqslant 3 / 2$. Conversely, if $a>3 / 2$, then $\pi^{*} L-a E$ is not nef, because $\left(\pi^{*} L-a E\right)\left(\pi^{*} L-2 E\right)=3-2 a<0$ and $\pi^{*} L-2 E$ is the class of the proper transform of $C_{x}$. Thus, $\epsilon_{x}(L) \geqslant 3 / 2$, and so $\epsilon_{x}(L)=3 / 2$, and $C_{x}$ is a Seshadri curve for $x$ with respect to $L$.

We now turn to the computation of $\alpha$. The stable base locus of $\pi^{*} L-2 E$ is $\widetilde{C}_{x}$, the proper transform of $C_{x}$. Hence, by Theorem $3.3(\mathrm{~b})$, either $\alpha_{x}(L) \geqslant 2$ or $\alpha_{x}(L)=\alpha_{x, C_{x}}\left(\left.L\right|_{C_{x}}\right)$ (note that $d=1$ ). By intersecting $X$ with a hyperplane containing $x$ and one of the lines, we produce a $k$-rational conic passing through $x$, and approximating on the conic gives us $2 \geqslant \alpha_{x}(L)$. We therefore conclude that $\alpha_{x}(L)=\min \left(2, \alpha_{x, C_{x}}\left(\left.L\right|_{C_{x}}\right)\right)$.

The curve $C_{x}$ is singular at $x$, and since $x$ does not lie on a line, $C_{x}$ is also irreducible. In particular, $C_{x}$ is an irreducible curve of geometric genus zero, and since $x$ is defined over $k, C_{x}$ is birational to $\mathbb{P}^{1}$ over $k$, via projection from $x$ in the tangent plane.

Applying Theorem 2.8 to $C_{x}$, we find that

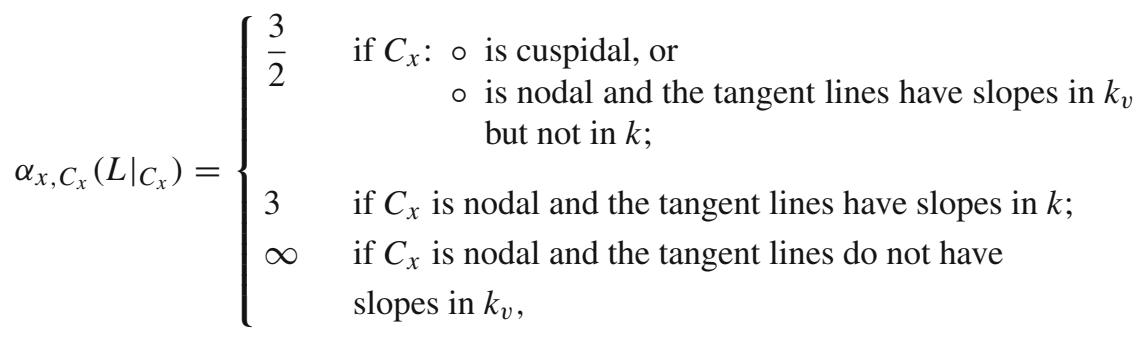


and this implies the stated values of $\alpha_{x}(L)$ above.

We now treat the case of a general nef divisor $D$. In what follows, we assume that the point $x$ does not lie on a $(-1)$-curve on $X$. We begin with a calculation of the Seshadri constant $\epsilon$. To do this, we will need some notation.

Let $\varphi: X \rightarrow \mathbb{P}^{2}$ be the blowing down map, and let $E_{1}, \ldots, E_{6}$ be the exceptional divisors of $\varphi$. We define the following linear equivalence classes on $X$ :

- $L=\varphi^{*} \mathcal{O}(1)$,

- $L_{i}=L-E_{i}$, the strict transform of a line through $P_{i}=\varphi\left(E_{i}\right)$,

- $L_{i j}=2 L-\left(\sum E_{n}\right)+E_{i}+E_{j}$ (where $\left.i \neq j\right)$, the strict transform of a conic through the four points $P_{n}$ with $n \neq i, j$,

- $B_{i}=3 L-\left(\sum E_{n}\right)-E_{i}$, the strict transform of a cubic curve through all six points $P_{n}$, with a node at $P_{i}$.

Let $h$ be the class of a hyperplane in the anticanonical embedding $X \subset \mathbb{P}^{3}$. For any line $\ell$ on $X$, the hyperplanes containing $\ell$ give (after removing $\ell$ ) a base-point-free pencil on $X$. If $x \in X$ does not lie on a line then the unique curve in this pencil through $x$ is smooth and irreducible. The classes $\left\{L_{i}, L_{i j}, B_{i}\right\}$ defined above are the 27 pencils coming from the lines. Recall that for any point $x$ on $X$ we use $C_{x}$ for the intersection of $X$ with its tangent plane at $x$ (so $C_{x}$ has class $h$ ). If $x$ does not lie on a line, then $C_{x}$ is a plane cubic curve with one double point, at $x$.

Theorem 4.6 Let $x$ be a point on $X$ that does not lie on a $(-1)$-curve, and let $D$ be a nef divisor on $X$. The Seshadri constant $\epsilon_{x}(D)$ is equal to $\min \left\{D \cdot L_{i}, D \cdot L_{i j}, D \cdot B_{i}\right.$, $(D \cdot h) / 2\}$.

Proof The nef cone $\Gamma$ of $X$ has 99 generators, which are listed in Sect. 5, Table 1. Let $S$ be the set of 27 divisor classes $\left\{L_{i}, L_{i j}, B_{i}\right\}$ as $i$ and $j$ range over all possible values, and for each element $C$ in $S$, we define the subcone $\Gamma(C)$ by

$$
\Gamma(C)=\left\{D \in \Gamma: D \cdot C=\min _{C^{\prime} \in S}\left\{D \cdot C^{\prime}\right\} \text { and } D \cdot C \leqslant(D \cdot h) / 2\right\} .
$$

Further define the subcone $\Gamma(h)$ to be

$$
\Gamma(h)=\left\{D \in \Gamma:(D \cdot h) / 2 \leqslant \min _{C^{\prime} \in S}\left\{D \cdot C^{\prime}\right\}\right\} .
$$

It is clear that $\Gamma$ is the union of these 28 subcones. To prove Theorem 4.6, it suffices to show that for every subcone $\Gamma(C)$, with $C \in S$, the curve through $x$ in the pencil corresponding to $C$ is a Seshadri curve for $x$ with respect to $D$ for all $D \in \Gamma(C)$ (respectively, in the case of the subcone $\Gamma(h)$, that $C_{x}$ is a Seshadri curve for $x$ with respect to $D$ for all $D \in \Gamma(h)$ ). By Proposition 4.4(b), it further suffices to prove this for $D$ a generator of the cone $\Gamma(C)$ (respectively $\Gamma(h)$ ).

The fundamental group of the space of all smooth cubic surfaces acts via monodromy on the Néron-Severi lattice of $X$. This monodromy action preserves the hyperplane class $h$ and acts transitively on the classes of the lines. Thus, up to monodromy action, there are only two of these subcones: $\Gamma\left(L_{1}\right)$ and $\Gamma(h)$. Generators for each of these subcones can be found in Sect. 5. Let $F=F_{x, L_{1}}$ be the 
unique curve in the pencil $L_{1}$ passing through $x$. For each generator $D$ of $\Gamma\left(L_{1}\right)$, it is straightforward to verify that $F$ is a Seshadri curve for $x$ with respect to $D$. These verifications also appear in Sect. 5. Each generator $G$ of $\Gamma(h)$ is also a generator of one of the other twenty-seven subcones $\Gamma(C)$, and for each such $G$, we have $G \cdot C=(G \cdot h) / 2=\left(G \cdot C_{x}\right) /$ mult $_{x}\left(C_{x}\right)$. Thus, since $C$ is a Seshadri curve for $x$ with respect to $G$, it follows that $C_{x}$ is also a Seshadri curve for $x$ with respect to $G$, and so $C_{x}$ is a Seshadri curve for every element of $\Gamma(h)$.

The next step is to calculate $\alpha_{x}$ for a point on a cubic surface.

Theorem 4.7 Let $x \in X(k)$ be a point that does not lie on a $(-1)$-curve, and let $D$ be a nef divisor on $X$. If the tangent curve $C_{x}$ is a cuspidal cubic, or a nodal cubic whose tangent lines at $x$ are defined over $k_{v}$ but not defined over $k$, then $\alpha_{x}(D)=\epsilon_{x}(D)$. Otherwise, $\alpha_{x}(D)=\min \left\{D \cdot L_{i}, D \cdot L_{i j}, D \cdot B_{i}\right\}$.

Proof Suppose that $D$ is in one of the cones $\Gamma(C)$ for $C \in S$, and let $F_{x, C}$ be the element of the pencil corresponding to $C$ which passes through $x$. Since $F_{x, C}$ is a smooth $k$-rational curve, we have

$$
D \cdot C \stackrel{\text { Theorem } 2.8}{=} \alpha_{x}\left(\left.D\right|_{F_{x, C}}\right) \stackrel{\text { Proposition 2.11(c) }}{\geqslant} \alpha_{x}(D) \stackrel{\text { Corollary } 3.5}{\geqslant} \epsilon_{x}(D) \stackrel{\text { Theorem }}{=}{ }^{.6} D \cdot C .
$$

(Note that the blowup of $X$ at a point not on a line is a del Pezzo surface of degree two, and so every nef divisor on the blowup is semiample (see [1, Theorem 5.1.2.1]), and thus has nonempty stable base locus. In particular the hypotheses of Corollary 3.5 are satisfied.) Thus $\alpha_{x}(D)=D \cdot C$ and $F_{x, C}$ is a curve of best approximation with respect to $D$.

Now suppose that $D \in \Gamma(h)$. If $C_{x}$ is cuspidal, or nodal with tangent lines having slopes in $k_{v}$ but not $k$, then Theorem 2.8 gives $\alpha_{x}\left(\left.D\right|_{C_{x}}\right)=D \cdot C_{x} / 2=$ $D \cdot C_{x} /$ mult $_{x}\left(C_{x}\right)$. By Theorem 4.6, $\epsilon_{x}(D)=D \cdot C_{x} / 2$, and so as above we conclude that $\alpha_{x}(D)=D \cdot C_{x}=\epsilon_{x}(D)$, and that $C_{x}$ is a curve of best approximation for $D$.

We now assume that $C_{x}$ is nodal and the slopes of the tangent lines are in $k$ or not in $k_{v}$. The codimension one faces of $\Gamma(h)$ (i.e., the facets) occur where one of the inequalities defining $\Gamma(h)$ becomes an equality, so that each facet is the intersection of $\Gamma(h)$ and $\Gamma(C)$ for some $C \in S$. For each $C \in S$ set $\widehat{\Gamma}(C)$ to be the cone generated by $\Gamma(C)$ and $-K$. Since $-K$ is in the interior of $\Gamma(h)$ it follows that $\Gamma$ is the union of the $\widehat{\Gamma}(C), C \in S$.

As above, for any $C \in S$, let $F_{x, C}$ be the member of the pencil corresponding to $C$ passing through $x$. In the proof of Theorem 4.5 we have seen that $F_{x, C}$ is a curve of best approximation for $-K$, and in the first part of the argument above that $F_{x, C}$ is a curve of best approximation for all $D \in \Gamma(C)$. By Proposition 4.4 (a), we conclude that $F_{x, C}$ is a curve of best approximation for all $D \in \widehat{\Gamma}(C)$. The result follows.

Note that as part of the proof we have shown that Conjecture 4.2 holds for every point $x \in X$ not on a $(-1)$-curve. 


\section{Appendix A: Generators of nef cones and subcones for the cubic surface}

A version of this appendix, with additional tables and larger font, may be found at [11]. In all the tables below, the first column is a numerical identifier of the vector in that row. In Tables 1, 2, and 4 the subsequent columns represent the coefficients of the vector with respect to the basis $\left\{L, E_{1}, \ldots, E_{6}\right\}$ of the Néron-Severi group of $X$. Thus, vector number 1 in Table 1 is the divisor class $2 L-E_{1}-E_{2}-E_{3}$. Each of the cones has 99 generators. There is no correspondence or relation between the rows in Tables 1, 2, and 4 with the same numerical identifier.

Table 1, of generators of the nef cone, is reproducing information that has been well known for some time, of course. It was calculated for these tables by finding generators for the cone obtained as the intersection of the half-spaces corresponding to non-negative intersection with each of the 27 lines on the cubic surface.

Table 1 Generators of the nef cone $\Gamma$ of a smooth cubic surface

\begin{tabular}{rrrrrrrr}
\hline$\#$ & $L$ & $E_{1}$ & $E_{2}$ & $E_{3}$ & $E_{4}$ & $E_{5}$ & $E_{6}$ \\
\hline $\mathbf{1}$ & 2 & -1 & -1 & -1 & 0 & 0 & 0 \\
$\mathbf{2}$ & 2 & -1 & -1 & 0 & -1 & 0 & 0 \\
$\mathbf{3}$ & 2 & -1 & -1 & 0 & 0 & -1 & 0 \\
$\mathbf{4}$ & 2 & -1 & -1 & 0 & 0 & 0 & -1 \\
$\mathbf{5}$ & 2 & -1 & 0 & -1 & -1 & 0 & 0 \\
$\mathbf{6}$ & 2 & -1 & 0 & -1 & 0 & -1 & 0 \\
$\mathbf{7}$ & 2 & -1 & 0 & -1 & 0 & 0 & -1 \\
$\mathbf{8}$ & 2 & -1 & 0 & 0 & -1 & -1 & 0 \\
$\mathbf{9}$ & 2 & -1 & 0 & 0 & -1 & 0 & -1 \\
$\mathbf{1 0}$ & 2 & -1 & 0 & 0 & 0 & -1 & -1 \\
$\mathbf{1 1}$ & 1 & 0 & 0 & 0 & 0 & 0 & 0 \\
$\mathbf{1 2}$ & 3 & -2 & -1 & -1 & -1 & -1 & 0 \\
$\mathbf{1 3}$ & 3 & -2 & -1 & -1 & -1 & 0 & -1 \\
$\mathbf{1 4}$ & 3 & -2 & -1 & -1 & 0 & -1 & -1 \\
$\mathbf{1 5}$ & 3 & -2 & -1 & 0 & -1 & -1 & -1 \\
$\mathbf{1 6}$ & 3 & -2 & 0 & -1 & -1 & -1 & -1 \\
$\mathbf{1 7}$ & 1 & -1 & 0 & 0 & 0 & 0 & 0 \\
$\mathbf{1 8}$ & 1 & 0 & -1 & 0 & 0 & 0 & 0 \\
$\mathbf{1 9}$ & 1 & 0 & 0 & -1 & 0 & 0 & 0 \\
$\mathbf{2 0}$ & 1 & 0 & 0 & 0 & -1 & 0 & 0 \\
$\mathbf{2 1}$ & 1 & 0 & 0 & 0 & 0 & -1 & 0 \\
$\mathbf{2 2}$ & 1 & 0 & 0 & 0 & 0 & 0 & -1 \\
$\mathbf{2 3}$ & 2 & 0 & -1 & -1 & -1 & 0 & 0 \\
$\mathbf{2 4}$ & 2 & 0 & -1 & -1 & 0 & -1 & 0 \\
$\mathbf{2 5}$ & 2 & 0 & -1 & -1 & 0 & 0 & -1 \\
$\mathbf{2 6}$ & 2 & 0 & -1 & 0 & -1 & -1 & 0 \\
\hline & & & & & & &
\end{tabular}


Table 1 continued

\begin{tabular}{lccccccr}
\hline$\#$ & $L$ & $E_{1}$ & $E_{2}$ & $E_{3}$ & $E_{4}$ & $E_{5}$ & $E_{6}$ \\
\hline 27 & 2 & 0 & -1 & 0 & -1 & 0 & -1
\end{tabular}

$\begin{array}{rrrrrrrr}27 & 2 & 0 & -1 & 0 & -1 & 0 & - \\ 28 & 2 & 0 & -1 & 0 & 0 & -1 & -\end{array}$

$\begin{array}{lllllllll}29 & 2 & 0 & 0 & -1 & -1 & -1 & 0\end{array}$

$\begin{array}{llllllll}30 & 2 & 0 & 0 & -1 & -1 & 0 & -1\end{array}$

$\begin{array}{llllllll}31 & 2 & 0 & 0 & -1 & 0 & -1 & -1\end{array}$

$\begin{array}{llllllll}32 & 2 & 0 & 0 & 0 & -1 & -1 & -1\end{array}$

$\begin{array}{llllllll}33 & 2 & -1 & -1 & -1 & -1 & 0 & 0\end{array}$

$\begin{array}{llllllll}34 & 2 & -1 & -1 & -1 & 0 & -1 & 0\end{array}$

$\begin{array}{llllllll}35 & 2 & -1 & -1 & -1 & 0 & 0 & -1\end{array}$

$\begin{array}{llllllll}36 & 2 & -1 & -1 & 0 & -1 & -1 & 0\end{array}$

$\begin{array}{llllllll}37 & 2 & -1 & -1 & 0 & -1 & 0 & -1\end{array}$

$\begin{array}{llllllll}38 & 2 & -1 & -1 & 0 & 0 & -1 & -1\end{array}$

$\begin{array}{llllllll}39 & 2 & -1 & 0 & -1 & -1 & -1 & 0\end{array}$

$\begin{array}{llllllll}40 & 2 & -1 & 0 & -1 & -1 & 0 & -1\end{array}$

$\begin{array}{lllllllll}41 & 2 & -1 & 0 & -1 & 0 & -1 & -1\end{array}$

$\begin{array}{lllllllll}42 & 2 & -1 & 0 & 0 & -1 & -1 & -1\end{array}$

$\begin{array}{llllllll}43 & 2 & 0 & -1 & -1 & -1 & -1 & 0\end{array}$

$\begin{array}{lllllllll}44 & 2 & 0 & -1 & -1 & -1 & 0 & -1\end{array}$

$\begin{array}{llllllll}45 & 2 & 0 & -1 & -1 & 0 & -1 & -1\end{array}$

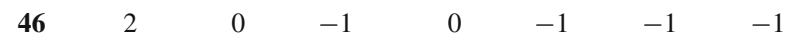

$\begin{array}{lllllllll}47 & 2 & 0 & 0 & -1 & -1 & -1 & -1\end{array}$

$\begin{array}{llllllll}48 & 3 & -1 & -2 & -1 & -1 & -1 & 0\end{array}$

$\begin{array}{llllllll}49 & 3 & -1 & -2 & -1 & -1 & 0 & -1\end{array}$

$\begin{array}{llllllll}50 & 3 & -1 & -2 & -1 & 0 & -1 & -1\end{array}$

$\begin{array}{llllllll}51 & 3 & -1 & -2 & 0 & -1 & -1 & -1\end{array}$

$\begin{array}{llllllll}52 & 3 & -1 & -1 & -2 & -1 & -1 & 0\end{array}$

$\begin{array}{llllllll}53 & 3 & -1 & -1 & -2 & -1 & 0 & -1\end{array}$

$\begin{array}{llllllll}54 & 3 & -1 & -1 & -2 & 0 & -1 & -1\end{array}$

$\begin{array}{llllllll}55 & 3 & -1 & -1 & -1 & -2 & -1 & 0\end{array}$

$\begin{array}{llllllll}56 & 3 & -1 & -1 & -1 & -2 & 0 & -1\end{array}$

$\begin{array}{llllllll}57 & 3 & -1 & -1 & -1 & -1 & -2 & 0\end{array}$

$\begin{array}{llllllll}58 & 3 & -1 & -1 & -1 & -1 & 0 & -2\end{array}$

$\begin{array}{llllllll}59 & 3 & -1 & -1 & -1 & 0 & -2 & -1\end{array}$

$60 \begin{array}{llllllll}6 & 3 & -1 & -1 & -1 & 0 & -1 & -2\end{array}$

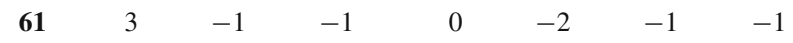

$\begin{array}{lllllllll}62 & 3 & -1 & -1 & 0 & -1 & -2 & -1\end{array}$

$\begin{array}{lllllllll}63 & 3 & -1 & -1 & 0 & -1 & -1 & -2\end{array}$

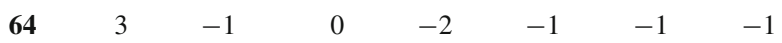

$\begin{array}{lllllllll}65 & 3 & -1 & 0 & -1 & -2 & -1 & -1\end{array}$

$66 \begin{array}{llllllll}6 & 3 & -1 & 0 & -1 & -1 & -2 & -1\end{array}$

$\begin{array}{llllllll}67 & 3 & -1 & 0 & -1 & -1 & -1 & -2\end{array}$ 
Table 1 continued

\begin{tabular}{lrrrrrrr}
\hline$\#$ & $L$ & $E_{1}$ & $E_{2}$ & $E_{3}$ & $E_{4}$ & $E_{5}$ & $E_{6}$ \\
\hline $\mathbf{6 8}$ & 3 & 0 & -2 & -1 & -1 & -1 & -1 \\
$\mathbf{6 9}$ & 3 & 0 & -1 & -2 & -1 & -1 & -1 \\
$\mathbf{7 0}$ & 3 & 0 & -1 & -1 & -2 & -1 & -1 \\
$\mathbf{7 1}$ & 3 & 0 & -1 & -1 & -1 & -2 & -1 \\
$\mathbf{7 2}$ & 3 & 0 & -1 & -1 & -1 & -1 & -2 \\
$\mathbf{7 3}$ & 3 & -2 & -1 & -1 & -1 & -1 & -1 \\
$\mathbf{7 4}$ & 3 & -1 & -2 & -1 & -1 & -1 & -1 \\
$\mathbf{7 5}$ & 3 & -1 & -1 & -2 & -1 & -1 & -1 \\
$\mathbf{7 6}$ & 3 & -1 & -1 & -1 & -2 & -1 & -1 \\
$\mathbf{7 7}$ & 3 & -1 & -1 & -1 & -1 & -2 & -1 \\
$\mathbf{7 8}$ & 3 & -1 & -1 & -1 & -1 & -1 & -2 \\
$\mathbf{7 9}$ & 4 & -2 & -2 & -2 & -1 & -1 & -1 \\
$\mathbf{8 0}$ & 4 & -2 & -2 & -1 & -2 & -1 & -1 \\
$\mathbf{8 1}$ & 4 & -2 & -2 & -1 & -1 & -2 & -1 \\
$\mathbf{8 2}$ & 4 & -2 & -2 & -1 & -1 & -1 & -2 \\
$\mathbf{8 3}$ & 4 & -2 & -1 & -2 & -2 & -1 & -1 \\
$\mathbf{8 4}$ & 4 & -2 & -1 & -2 & -1 & -2 & -1 \\
$\mathbf{8 5}$ & 4 & -2 & -1 & -2 & -1 & -1 & -2 \\
$\mathbf{8 6}$ & 4 & -2 & -1 & -1 & -2 & -2 & -1 \\
$\mathbf{8 7}$ & 4 & -2 & -1 & -1 & -2 & -1 & -2 \\
$\mathbf{8 8}$ & 4 & -2 & -1 & -1 & -1 & -2 & -2 \\
$\mathbf{8 9}$ & 4 & -1 & -2 & -2 & -2 & -1 & -1 \\
$\mathbf{9 0}$ & 4 & -1 & -2 & -2 & -1 & -2 & -1 \\
$\mathbf{9 1}$ & 4 & -1 & -2 & -2 & -1 & -1 & -2 \\
$\mathbf{9 2}$ & 4 & -1 & -2 & -1 & -2 & -2 & -1 \\
$\mathbf{9 3}$ & 4 & -1 & -2 & -1 & -2 & -1 & -2 \\
$\mathbf{9 4}$ & 4 & -1 & -2 & -1 & -1 & -2 & -2 \\
$\mathbf{9 5}$ & 4 & -1 & -1 & -2 & -2 & -2 & -1 \\
$\mathbf{9 6}$ & 4 & -1 & -1 & -2 & -2 & -1 & -2 \\
$\mathbf{9 7}$ & 4 & -1 & -1 & -2 & -1 & -2 & -2 \\
$\mathbf{9 8}$ & 4 & -1 & -1 & -1 & -2 & -2 & -2 \\
$\mathbf{9 9}$ & 5 & -2 & -2 & -2 & -2 & -2 & -2 \\
\hline & & & & & & &
\end{tabular}

Table 2, of generators of the cone $\Gamma\left(L_{1}\right)$, was generated by using the half-spaces defining $\Gamma$ in addition to the half-spaces corresponding to the conditions $D \cdot L_{1}=$ $\min _{C^{\prime} \in S}\left\{D \cdot C^{\prime}\right\}$ and $D \cdot L_{1} \leqslant(D \cdot h) / 2$ for all $D \in \Gamma$.

We use $D_{n}$ to refer to the divisor class represented by row $n$ of Table 2 . For any point $x \in X$ not on a $(-1)$-curve, the unique curve $F=F_{x, L_{1}}$ in the pencil $L_{1}$ passing through $x$ is smooth and irreducible. Row $n$ in Table 3 below is a-very brief! justification of why $F$ is a Seshadri curve for $x$ with respect to $D_{n}$. 
Table 2 Generators $D_{n}$ of the cone $\Gamma\left(L_{1}\right)$

\begin{tabular}{|c|c|c|c|c|c|c|c|}
\hline$\#$ & $L$ & $E_{1}$ & $E_{2}$ & $E_{3}$ & $E_{4}$ & $E_{5}$ & $E_{6}$ \\
\hline 1 & 4 & -3 & -1 & -1 & -1 & -1 & -1 \\
\hline 2 & 2 & -1 & -1 & 0 & 0 & 0 & 0 \\
\hline 3 & 2 & -1 & 0 & -1 & 0 & 0 & 0 \\
\hline 4 & 2 & -1 & 0 & 0 & -1 & 0 & 0 \\
\hline 5 & 2 & -1 & 0 & 0 & 0 & -1 & 0 \\
\hline 6 & 2 & -1 & 0 & 0 & 0 & 0 & -1 \\
\hline 7 & 1 & 0 & 0 & 0 & 0 & 0 & 0 \\
\hline 8 & 3 & -2 & -1 & -1 & -1 & 0 & 0 \\
\hline 9 & 3 & -2 & -1 & -1 & 0 & -1 & 0 \\
\hline 10 & 3 & -2 & -1 & -1 & 0 & 0 & -1 \\
\hline 11 & 3 & -2 & -1 & 0 & -1 & -1 & 0 \\
\hline 12 & 3 & -2 & -1 & 0 & -1 & 0 & -1 \\
\hline 13 & 3 & -2 & -1 & 0 & 0 & -1 & -1 \\
\hline 14 & 3 & -2 & 0 & -1 & -1 & -1 & 0 \\
\hline 15 & 3 & -2 & 0 & -1 & -1 & 0 & -1 \\
\hline 16 & 3 & -2 & 0 & -1 & 0 & -1 & -1 \\
\hline 17 & 3 & -2 & 0 & 0 & -1 & -1 & -1 \\
\hline 18 & 1 & -1 & 0 & 0 & 0 & 0 & 0 \\
\hline 19 & 2 & -1 & -1 & -1 & 0 & 0 & 0 \\
\hline 20 & 2 & -1 & -1 & 0 & -1 & 0 & 0 \\
\hline 21 & 2 & -1 & -1 & 0 & 0 & -1 & 0 \\
\hline 22 & 2 & -1 & -1 & 0 & 0 & 0 & -1 \\
\hline 23 & 2 & -1 & 0 & -1 & -1 & 0 & 0 \\
\hline 24 & 2 & -1 & 0 & -1 & 0 & -1 & 0 \\
\hline 25 & 2 & -1 & 0 & -1 & 0 & 0 & -1 \\
\hline 26 & 2 & -1 & 0 & 0 & -1 & -1 & 0 \\
\hline 27 & 2 & -1 & 0 & 0 & -1 & 0 & -1 \\
\hline 28 & 2 & -1 & 0 & 0 & 0 & -1 & -1 \\
\hline 29 & 3 & -1 & -1 & -1 & -1 & 0 & 0 \\
\hline 30 & 3 & -1 & -1 & -1 & 0 & -1 & 0 \\
\hline 31 & 3 & -1 & -1 & -1 & 0 & 0 & -1 \\
\hline 32 & 3 & -1 & -1 & 0 & -1 & -1 & 0 \\
\hline 33 & 3 & -1 & -1 & 0 & -1 & 0 & -1 \\
\hline 34 & 3 & -1 & -1 & 0 & 0 & -1 & -1 \\
\hline 35 & 3 & -1 & 0 & -1 & -1 & -1 & 0 \\
\hline 36 & 3 & -1 & 0 & -1 & -1 & 0 & -1 \\
\hline 37 & 3 & -1 & 0 & -1 & 0 & -1 & -1 \\
\hline 38 & 3 & -1 & 0 & 0 & -1 & -1 & -1 \\
\hline 39 & 3 & -1 & -1 & -1 & -1 & -1 & 0 \\
\hline 40 & 3 & -1 & -1 & -1 & -1 & 0 & -1 \\
\hline 41 & 3 & -1 & -1 & -1 & 0 & -1 & -1 \\
\hline
\end{tabular}


Table 2 continued

\begin{tabular}{lrrrrrrr}
\hline$\#$ & $L$ & $E_{1}$ & $E_{2}$ & $E_{3}$ & $E_{4}$ & $E_{5}$ & $E_{6}$ \\
\hline $\mathbf{4 2}$ & 3 & -1 & -1 & 0 & -1 & -1 & -1
\end{tabular}

$\begin{array}{rrrrrrrr}42 & 3 & -1 & -1 & 0 & -1 & -1 & - \\ 43 & 3 & -1 & 0 & -1 & -1 & -1 & -\end{array}$

$\begin{array}{llllllll}44 & 4 & -1 & -1 & -1 & -1 & -1 & -1\end{array}$

$\begin{array}{llllllll}45 & 3 & -2 & -1 & -1 & -1 & -1 & 0\end{array}$

$\begin{array}{llllllll}46 & 3 & -2 & -1 & -1 & -1 & 0 & -1\end{array}$

$\begin{array}{llllllll}47 & 3 & -2 & -1 & -1 & 0 & -1 & -1\end{array}$

$\begin{array}{lllllllll}48 & 3 & -2 & -1 & 0 & -1 & -1 & -1\end{array}$

$\begin{array}{llllllll}49 & 3 & -2 & 0 & -1 & -1 & -1 & -1\end{array}$

$\begin{array}{llllllll}\mathbf{5 0} & 4 & -2 & -2 & -1 & -1 & -1 & 0\end{array}$

$\begin{array}{llllllll}\mathbf{5 1} & 4 & -2 & -2 & -1 & -1 & 0 & -1\end{array}$

$\begin{array}{llllllll}52 & 4 & -2 & -2 & -1 & 0 & -1 & -1\end{array}$

$\begin{array}{lllllllll}\mathbf{5 3} & 4 & -2 & -2 & 0 & -1 & -1 & -1\end{array}$

$\begin{array}{llllllll}54 & 4 & -2 & -1 & -2 & -1 & -1 & 0\end{array}$

$\begin{array}{llllllll}55 & 4 & -2 & -1 & -2 & -1 & 0 & -1\end{array}$

$\begin{array}{llllllll}56 & 4 & -2 & -1 & -2 & 0 & -1 & -1\end{array}$

$\begin{array}{llllllll}57 & 4 & -2 & -1 & -1 & -2 & -1 & 0\end{array}$

$\begin{array}{llllllll}58 & 4 & -2 & -1 & -1 & -2 & 0 & -1\end{array}$

$\begin{array}{llllllll}59 & 4 & -2 & -1 & -1 & -1 & -2 & 0\end{array}$

$\begin{array}{llllllll}60 & 4 & -2 & -1 & -1 & -1 & 0 & -2\end{array}$

$\begin{array}{llllllll}61 & 4 & -2 & -1 & -1 & 0 & -2 & -1\end{array}$

$\begin{array}{llllllll}62 & 4 & -2 & -1 & -1 & 0 & -1 & -2\end{array}$

$\begin{array}{llllllll}63 & 4 & -2 & -1 & 0 & -2 & -1 & -1\end{array}$

$\begin{array}{llllllll}64 & 4 & -2 & -1 & 0 & -1 & -2 & -1\end{array}$

$\begin{array}{llllllll}65 & 4 & -2 & -1 & 0 & -1 & -1 & -2\end{array}$

$\begin{array}{llllllll}66 & 4 & -2 & 0 & -2 & -1 & -1 & -1\end{array}$

$\begin{array}{llllllll}67 & 4 & -2 & 0 & -1 & -2 & -1 & -1\end{array}$

$\begin{array}{llllllll}68 & 4 & -2 & 0 & -1 & -1 & -2 & -1\end{array}$

$69 \begin{array}{llllllll}4 & -2 & 0 & -1 & -1 & -1 & -2\end{array}$

$\begin{array}{llllllll}70 & 4 & -2 & -2 & -1 & -1 & -1 & -1\end{array}$

$\begin{array}{llllllll}71 & 4 & -2 & -1 & -2 & -1 & -1 & -1\end{array}$

$\begin{array}{llllllll}72 & 4 & -2 & -1 & -1 & -2 & -1 & -1\end{array}$

$\begin{array}{llllllll}73 & 4 & -2 & -1 & -1 & -1 & -2 & -1\end{array}$

$\begin{array}{llllllll}74 & 4 & -2 & -1 & -1 & -1 & -1 & -2\end{array}$

$\begin{array}{llllllll}75 & 5 & -2 & -2 & -2 & -1 & -1 & -1\end{array}$

$\begin{array}{llllllll}76 & 5 & -2 & -2 & -1 & -2 & -1 & -1\end{array}$

$\begin{array}{llllllll}77 & 5 & -2 & -2 & -1 & -1 & -2 & -1\end{array}$

$\begin{array}{llllllll}78 & 5 & -2 & -2 & -1 & -1 & -1 & -2\end{array}$

$\begin{array}{llllllll}79 & 5 & -2 & -1 & -2 & -2 & -1 & -1\end{array}$

$\begin{array}{llllllll}80 & 5 & -2 & -1 & -2 & -1 & -2 & -1\end{array}$

$\begin{array}{llllllll}81 & 5 & -2 & -1 & -2 & -1 & -1 & -2\end{array}$

$\begin{array}{llllllll}82 & 5 & -2 & -1 & -1 & -2 & -2 & -1\end{array}$


Table 2 continued

\begin{tabular}{llllllll}
\hline$\#$ & $L$ & $E_{1}$ & $E_{2}$ & $E_{3}$ & $E_{4}$ & $E_{5}$ & $E_{6}$ \\
\hline $\mathbf{8 3}$ & 5 & -2 & -1 & -1 & -2 & -1 & -2 \\
$\mathbf{8 4}$ & 5 & -2 & -1 & -1 & -1 & -2 & -2 \\
$\mathbf{8 5}$ & 5 & -3 & -2 & -2 & -1 & -1 & -1 \\
$\mathbf{8 6}$ & 5 & -3 & -2 & -1 & -2 & -1 & -1 \\
$\mathbf{8 7}$ & 5 & -3 & -2 & -1 & -1 & -2 & -1 \\
$\mathbf{8 8}$ & 5 & -3 & -2 & -1 & -1 & -1 & -2 \\
$\mathbf{8 9}$ & 5 & -3 & -1 & -2 & -2 & -1 & -1 \\
$\mathbf{9 0}$ & 5 & -3 & -1 & -2 & -1 & -2 & -1 \\
$\mathbf{9 1}$ & 5 & -3 & -1 & -2 & -1 & -1 & -2 \\
$\mathbf{9 2}$ & 5 & -3 & -1 & -1 & -2 & -2 & -1 \\
$\mathbf{9 3}$ & 5 & -3 & -1 & -1 & -2 & -1 & -2 \\
$\mathbf{9 4}$ & 5 & -3 & -1 & -1 & -1 & -2 & -2 \\
$\mathbf{9 5}$ & 6 & -3 & -2 & -2 & -2 & -2 & -1 \\
$\mathbf{9 6}$ & 6 & -3 & -2 & -2 & -2 & -1 & -2 \\
$\mathbf{9 7}$ & 6 & -3 & -2 & -2 & -1 & -2 & -2 \\
$\mathbf{9 8}$ & 6 & -3 & -2 & -1 & -2 & -2 & -2 \\
$\mathbf{9 9}$ & 6 & -3 & -1 & -2 & -2 & -2 & -2 \\
\hline
\end{tabular}

As an example, in row 1 of Table 3, the "Reason" is $L_{1} \cdot D_{1}=1$, and thus $F \cdot D_{1}=L_{1} \cdot D_{1}=1$. We claim that for the divisors $D_{n}, \epsilon_{x}$ is always at least one if it is nonzero. Furthermore, if $n \neq 18$ then $\epsilon_{x}\left(D_{n}\right) \geqslant 1$ for $x$ not on a $(-1)$-curve. Granting these claims, since by assumption, $x$ does not lie on any (-1)curve (and since $D_{1} \neq D_{18}$ ), we have $\epsilon_{x}\left(D_{1}\right) \geqslant 1$. The curve $F$ is smooth at $x$, and the reason given tells us that $F$ has degree 1 with respect to $D_{1}$. Therefore $\left(D_{1} \cdot F\right) / \operatorname{mult}_{x}(F)=1 / 1=1$, hence $\epsilon_{x}\left(D_{1}\right)=1$, and $F$ is a Seshadri curve for $x$ with respect to $D_{1}$.

To see the claims, notice that the generators of the nef cone $\Gamma$ (see Table 1) are all either morphisms to $\mathbb{P}^{1}$ corresponding to pencils of conics on the cubic surface, or else morphisms to $\mathbb{P}^{2}$ that are the blowing down of six pairwise disjoint $(-1)$-curves. For a point $x$ not on a $(-1)$-curve $\epsilon_{x}$ is at least one for the blowdowns to $\mathbb{P}^{2}$, and $\epsilon_{x}=0$ for the pencils no matter which point $x$ is. It is straightforward to check that all the generators listed in Table 2 are non-negative integer linear combinations of the generators of the nef cones, and therefore (by Proposition 2.11 (b)) enjoy the same property: for any point $x$, the Seshadri constant $\epsilon_{x}\left(D_{i}\right)$ is either zero or else is at least one. Furthermore the only generator of $\Gamma\left(L_{1}\right)$ which is morphism to $\mathbb{P}^{1}$ is $D_{18}=L_{1}$, and thus we have $\epsilon_{x}\left(D_{n}\right) \geqslant 1$ for all $n \neq 18$.

Similar arguments explain other reasons of the form " $D_{n} \cdot L_{1}=1$ " or " $=0$ ".

As a second example of a reason, in row 29 of Table 3, the comment " $L+L_{56}$ " means that the divisor $D_{29}$ represented by that row is the sum of $L$ and $L_{56}$. Again 
Table 3 Reasons $F$ is a Seshadri curve for $D_{n}$

\begin{tabular}{|c|c|}
\hline$\#$ & Reason \\
\hline 1 & $L_{1} \cdot D_{1}=1$ \\
\hline 2 & $L_{1} \cdot D_{2}=1$ \\
\hline 3 & $L_{1} \cdot D_{3}=1$ \\
\hline 4 & $L_{1} \cdot D_{4}=1$ \\
\hline 5 & $L_{1} \cdot D_{5}=1$ \\
\hline 6 & $L_{1} \cdot D_{6}=1$ \\
\hline 7 & $L_{1} \cdot D_{7}=1$ \\
\hline 8 & $L_{1} \cdot D_{8}=1$ \\
\hline 9 & $L_{1} \cdot D_{9}=1$ \\
\hline 10 & $L_{1} \cdot D_{10}=1$ \\
\hline 11 & $L_{1} \cdot D_{11}=1$ \\
\hline 12 & $L_{1} \cdot D_{12}=1$ \\
\hline 13 & $L_{1} \cdot D_{13}=1$ \\
\hline 14 & $L_{1} \cdot D_{14}=1$ \\
\hline 15 & $L_{1} \cdot D_{15}=1$ \\
\hline 16 & $L_{1} \cdot D_{16}=1$ \\
\hline 17 & $L_{1} \cdot D_{17}=1$ \\
\hline 18 & $L_{1} \cdot D_{18}=0$ \\
\hline 19 & $L_{1} \cdot D_{19}=1$ \\
\hline 20 & $L_{1} \cdot D_{20}=1$ \\
\hline 21 & $L_{1} \cdot D_{21}=1$ \\
\hline 22 & $L_{1} \cdot D_{22}=1$ \\
\hline 23 & $L_{1} \cdot D_{23}=1$ \\
\hline 24 & $L_{1} \cdot D_{24}=1$ \\
\hline 25 & $L_{1} \cdot D_{25}=1$ \\
\hline 26 & $L_{1} \cdot D_{26}=1$ \\
\hline 27 & $L_{1} \cdot D_{27}=1$ \\
\hline 28 & $L_{1} \cdot D_{28}=1$ \\
\hline 29 & $L+L_{56}$ \\
\hline 30 & $L+L_{46}$ \\
\hline 31 & $L+L_{45}$ \\
\hline 32 & $L+L_{36}$ \\
\hline 33 & $L+L_{35}$ \\
\hline 34 & $L+L_{34}$ \\
\hline 35 & $L+L_{26}$ \\
\hline 36 & $L+L_{25}$ \\
\hline 37 & $L+L_{24}$ \\
\hline 38 & $L+L_{23}$ \\
\hline 39 & $L_{2}+L_{26}$ \\
\hline 40 & $L_{2}+L_{25}$ \\
\hline 41 & $L_{2}+L_{24}$ \\
\hline
\end{tabular}


Table 3 continued

\begin{tabular}{|c|c|}
\hline$\#$ & Reason \\
\hline 42 & $L_{2}+L_{23}$ \\
\hline 43 & $L_{3}+L_{23}$ \\
\hline 44 & $L_{23}+L_{2}+L_{3}$ \\
\hline 45 & $L_{1} \cdot D_{45}=1$ \\
\hline 46 & $L_{1} \cdot D_{46}=1$ \\
\hline 47 & $L_{1} \cdot D_{47}=1$ \\
\hline 48 & $L_{1} \cdot D_{48}=1$ \\
\hline 49 & $L_{1} \cdot D_{49}=1$ \\
\hline 50 & $D_{45}+L_{2}$ \\
\hline 51 & $D_{46}+L_{2}$ \\
\hline 52 & $D_{47}+L_{2}$ \\
\hline 53 & $D_{48}+L_{2}$ \\
\hline 54 & $D_{45}+L_{3}$ \\
\hline 55 & $D_{46}+L_{3}$ \\
\hline 56 & $D_{47}+L_{3}$ \\
\hline 57 & $D_{45}+L_{4}$ \\
\hline 58 & $D_{46}+L_{4}$ \\
\hline 59 & $D_{45}+L_{5}$ \\
\hline 60 & $D_{46}+L_{6}$ \\
\hline 61 & $D_{47}+L_{5}$ \\
\hline 62 & $D_{47}+L_{6}$ \\
\hline 63 & $D_{48}+L_{4}$ \\
\hline 64 & $D_{48}+L_{5}$ \\
\hline 65 & $D_{48}+L_{6}$ \\
\hline 66 & $D_{49}+L_{3}$ \\
\hline 67 & $D_{49}+L_{4}$ \\
\hline 68 & $D_{49}+L_{5}$ \\
\hline 69 & $D_{49}+L_{6}$ \\
\hline 70 & $B_{1}+L_{2}$ \\
\hline 71 & $B_{1}+L_{3}$ \\
\hline 72 & $B_{1}+L_{4}$ \\
\hline 73 & $B_{1}+L_{5}$ \\
\hline 74 & $B_{1}+L_{6}$ \\
\hline 75 & $L_{3}+L_{34}+L_{56}$ \\
\hline 76 & $L_{2}+L_{23}+L_{56}$ \\
\hline 77 & $L_{2}+L_{23}+L_{46}$ \\
\hline 78 & $L_{2}+L_{23}+L_{45}$ \\
\hline 79 & $L_{3}+L_{23}+L_{56}$ \\
\hline 80 & $L_{3}+L_{23}+L_{46}$ \\
\hline 81 & $L_{3}+L_{23}+L_{45}$ \\
\hline 82 & $L_{4}+L_{23}+L_{46}$ \\
\hline
\end{tabular}


Table 3 continued

\begin{tabular}{ll}
\hline$\#$ & Reason \\
\hline $\mathbf{8 3}$ & $L_{4}+L_{23}+L_{45}$ \\
$\mathbf{8 4}$ & $L_{5}+L_{23}+L_{45}$ \\
$\mathbf{8 5}$ & $D_{45}+L_{45}$ \\
$\mathbf{8 6}$ & $D_{45}+L_{35}$ \\
$\mathbf{8 7}$ & $D_{45}+L_{34}$ \\
$\mathbf{8 8}$ & $D_{46}+L_{34}$ \\
$\mathbf{8 9}$ & $D_{45}+L_{25}$ \\
$\mathbf{9 0}$ & $D_{45}+L_{24}$ \\
$\mathbf{9 1}$ & $D_{46}+L_{24}$ \\
$\mathbf{9 2}$ & $D_{45}+L_{23}$ \\
$\mathbf{9 3}$ & $D_{46}+L_{23}$ \\
$\mathbf{9 4}$ & $D_{47}+L_{23}$ \\
$\mathbf{9 5}$ & $L_{23}+L_{46}+L_{56}$ \\
$\mathbf{9 6}$ & $L_{23}+L_{45}+L_{56}$ \\
$\mathbf{9 7}$ & $L_{23}+L_{45}+L_{46}$ \\
$\mathbf{9 8}$ & $L_{23}+L_{34}+L_{56}$ \\
$\mathbf{9 9}$ & $L_{23}+L_{24}+L_{56}$ \\
\hline
\end{tabular}

suppose that $x$ does not lie on a $(-1)$-curve. Any curve $C$ passing through $x$ that has nonzero intersection with $L$ must have $L \cdot C /$ mult $_{x}(C) \geqslant 1$, since $L$ is an isomorphism away from $(-1)$-curves. Similarly, any curve not contracted by $L_{56}$ must also satisfy $L_{56} \cdot C / \operatorname{mult}_{x}(C) \geqslant 1$, so any curve not contracted by $L_{56}$ or $L$ must satisfy $\left(L+L_{56}\right) \cdot C / \operatorname{mult}_{x}(C) \geqslant 2$. If $C$ is contracted by $L_{56}$, then it is either a $(-1)$ curve, or else it is an element of the divisor class $L_{56}$ itself, in which case it satisfies $\left(L+L_{56}\right) \cdot C / \operatorname{mult}_{x}(C)=2$ by direct calculation. In all cases, since $x$ does not lie on a $(-1)$-curve, we see that $\epsilon_{x}\left(L+L_{56}\right) \geqslant 2$. Since $L_{1} \cdot L=L_{1} \cdot L_{56}=1$, we compute that $\left(L+L_{56}\right) \cdot F / \operatorname{mult}_{x}(F)=(1+1) / 1=2$ and therefore that $\epsilon_{x}\left(L+L_{56}\right)=2$. Hence $F$ is a Seshadri curve for $x$ with respect to $D_{29}=L+L_{56}$. Similar arguments explain the other reasons of the form " $a+b$ " or " $a+b+c$ ".

For these types of arguments, it is useful to know that $L_{1}$ (and hence $F$ ) has intersection number one with the divisors $L, B_{1}, L_{i}$ for $i \neq 1$, and $L_{i j}$ for $i, j \neq 1$.

Table 4 , of generators of the cone $\Gamma(h)$, was generated by using the half-spaces defining $\Gamma$ in addition to the half-spaces corresponding to the intersection inequalities $(D \cdot h) / 2 \leqslant \min _{C^{\prime} \in S}\left\{D \cdot C^{\prime}\right\}$ for all $D \in \Gamma$.

We use $G_{n}$ to refer to the divisor class represented by row $n$ of Table 4. The rightmost column of row $n$ is a divisor class $C \in S$ such that $G_{n}$ is also a generator of the subcone $\Gamma(C)$. From the definition of the cones $\Gamma(C)$ and $\Gamma(h)$, this implies that $G_{n} \cdot C=\left(G_{n} \cdot h\right) / 2$. As explained in the proof of Theorem 4.7, this provides a verification that $C_{x}$ is a Seshadri curve for $x$ with respect to $G_{n}$. 
Table 4 Generators $G_{n}$ of the cone $\Gamma(h)$

\begin{tabular}{|c|c|c|c|c|c|c|c|c|}
\hline \# & $L$ & $E_{1}$ & $E_{2}$ & $E_{3}$ & $E_{4}$ & $E_{5}$ & $E_{6}$ & Div. class \\
\hline 1 & 8 & -3 & -3 & -3 & -3 & -3 & -3 & $B_{1}$ \\
\hline 2 & 4 & -1 & -1 & -1 & -1 & -1 & -1 & $L_{1}$ \\
\hline 3 & 4 & -2 & -2 & -1 & -1 & -1 & -1 & $L_{1}$ \\
\hline 4 & 4 & -2 & -1 & -2 & -1 & -1 & -1 & $L_{1}$ \\
\hline 5 & 4 & -2 & -1 & -1 & -2 & -1 & -1 & $L_{1}$ \\
\hline 6 & 4 & -2 & -1 & -1 & -1 & -2 & -1 & $L_{1}$ \\
\hline 7 & 4 & -2 & -1 & -1 & -1 & -1 & -2 & $L_{1}$ \\
\hline 8 & 4 & -1 & -2 & -2 & -1 & -1 & -1 & $L_{2}$ \\
\hline 9 & 4 & -1 & -2 & -1 & -2 & -1 & -1 & $L_{2}$ \\
\hline 10 & 4 & -1 & -2 & -1 & -1 & -2 & -1 & $L_{2}$ \\
\hline 11 & 4 & -1 & -2 & -1 & -1 & -1 & -2 & $L_{2}$ \\
\hline 12 & 4 & -1 & -1 & -2 & -2 & -1 & -1 & $L_{3}$ \\
\hline 13 & 4 & -1 & -1 & -2 & -1 & -2 & -1 & $L_{3}$ \\
\hline 14 & 4 & -1 & -1 & -2 & -1 & -1 & -2 & $L_{3}$ \\
\hline 15 & 4 & -1 & -1 & -1 & -2 & -2 & -1 & $L_{4}$ \\
\hline 16 & 4 & -1 & -1 & -1 & -2 & -1 & -2 & $L_{4}$ \\
\hline 17 & 4 & -1 & -1 & -1 & -1 & -2 & -2 & $L_{5}$ \\
\hline 18 & 5 & -2 & -2 & -2 & -1 & -1 & -1 & $L_{1}$ \\
\hline 19 & 5 & -2 & -2 & -1 & -2 & -1 & -1 & $L_{1}$ \\
\hline 20 & 5 & -2 & -2 & -1 & -1 & -2 & -1 & $L_{1}$ \\
\hline 21 & 5 & -2 & -2 & -1 & -1 & -1 & -2 & $L_{1}$ \\
\hline 22 & 5 & -2 & -1 & -2 & -2 & -1 & -1 & $L_{1}$ \\
\hline 23 & 5 & -2 & -1 & -2 & -1 & -2 & -1 & $L_{1}$ \\
\hline 24 & 5 & -2 & -1 & -2 & -1 & -1 & -2 & $L_{1}$ \\
\hline 25 & 5 & -2 & -1 & -1 & -2 & -2 & -1 & $L_{1}$ \\
\hline 26 & 5 & -2 & -1 & -1 & -2 & -1 & -2 & $L_{1}$ \\
\hline 27 & 5 & -2 & -1 & -1 & -1 & -2 & -2 & $L_{1}$ \\
\hline 28 & 5 & -1 & -2 & -2 & -2 & -1 & -1 & $L_{2}$ \\
\hline 29 & 5 & -1 & -2 & -2 & -1 & -2 & -1 & $L_{2}$ \\
\hline 30 & 5 & -1 & -2 & -2 & -1 & -1 & -2 & $L_{2}$ \\
\hline 31 & 5 & -1 & -2 & -1 & -2 & -2 & -1 & $L_{2}$ \\
\hline 32 & 5 & -1 & -2 & -1 & -2 & -1 & -2 & $L_{2}$ \\
\hline 33 & 5 & -1 & -2 & -1 & -1 & -2 & -2 & $L_{2}$ \\
\hline 34 & 5 & -1 & -1 & -2 & -2 & -2 & -1 & $L_{3}$ \\
\hline 35 & 5 & -1 & -1 & -2 & -2 & -1 & -2 & $L_{3}$ \\
\hline 36 & 5 & -1 & -1 & -2 & -1 & -2 & -2 & $L_{3}$ \\
\hline 37 & 5 & -1 & -1 & -1 & -2 & -2 & -2 & $L_{4}$ \\
\hline 38 & 7 & -3 & -3 & -3 & -2 & -2 & -2 & $B_{1}$ \\
\hline 39 & 7 & -3 & -3 & -2 & -3 & -2 & -2 & $B_{1}$ \\
\hline 40 & 7 & -3 & -3 & -2 & -2 & -3 & -2 & $B_{1}$ \\
\hline 41 & 7 & -3 & -3 & -2 & -2 & -2 & -3 & $B_{1}$ \\
\hline
\end{tabular}


Table 4 continued

\begin{tabular}{|c|c|c|c|c|c|c|c|c|}
\hline \# & $L$ & $E_{1}$ & $E_{2}$ & $E_{3}$ & $E_{4}$ & $E_{5}$ & $E_{6}$ & Div. class \\
\hline 42 & 7 & -3 & -2 & -3 & -3 & -2 & -2 & $B_{1}$ \\
\hline 43 & 7 & -3 & -2 & -3 & -2 & -3 & -2 & $B_{1}$ \\
\hline 44 & 7 & -3 & -2 & -3 & -2 & -2 & -3 & $B_{1}$ \\
\hline 45 & 7 & -3 & -2 & -2 & -3 & -3 & -2 & $B_{1}$ \\
\hline 46 & 7 & -3 & -2 & -2 & -3 & -2 & -3 & $B_{1}$ \\
\hline 47 & 7 & -3 & -2 & -2 & -2 & -3 & -3 & $B_{1}$ \\
\hline 48 & 7 & -2 & -3 & -3 & -3 & -2 & -2 & $B_{2}$ \\
\hline 49 & 7 & -2 & -3 & -3 & -2 & -3 & -2 & $B_{2}$ \\
\hline 50 & 7 & -2 & -3 & -3 & -2 & -2 & -3 & $B_{2}$ \\
\hline 51 & 7 & -2 & -3 & -2 & -3 & -3 & -2 & $B_{2}$ \\
\hline 52 & 7 & -2 & -3 & -2 & -3 & -2 & -3 & $B_{2}$ \\
\hline 53 & 7 & -2 & -3 & -2 & -2 & -3 & -3 & $B_{2}$ \\
\hline 54 & 7 & -2 & -2 & -3 & -3 & -3 & -2 & $B_{3}$ \\
\hline 55 & 7 & -2 & -2 & -3 & -3 & -2 & -3 & $B_{3}$ \\
\hline 56 & 7 & -2 & -2 & -3 & -2 & -3 & -3 & $B_{3}$ \\
\hline 57 & 7 & -2 & -2 & -2 & -3 & -3 & -3 & $B_{4}$ \\
\hline 58 & 6 & -3 & -2 & -2 & -2 & -2 & -1 & $B_{1}$ \\
\hline 59 & 6 & -3 & -2 & -2 & -2 & -1 & -2 & $B_{1}$ \\
\hline 60 & 6 & -3 & -2 & -2 & -1 & -2 & -2 & $B_{1}$ \\
\hline 61 & 6 & -3 & -2 & -1 & -2 & -2 & -2 & $B_{1}$ \\
\hline 62 & 6 & -3 & -1 & -2 & -2 & -2 & -2 & $B_{1}$ \\
\hline 63 & 6 & -2 & -3 & -2 & -2 & -2 & -1 & $B_{2}$ \\
\hline 64 & 6 & -2 & -3 & -2 & -2 & -1 & -2 & $B_{2}$ \\
\hline 65 & 6 & -2 & -3 & -2 & -1 & -2 & -2 & $B_{2}$ \\
\hline 66 & 6 & -2 & -3 & -1 & -2 & -2 & -2 & $B_{2}$ \\
\hline 67 & 6 & -2 & -2 & -3 & -2 & -2 & -1 & $B_{3}$ \\
\hline 68 & 6 & -2 & -2 & -3 & -2 & -1 & -2 & $B_{3}$ \\
\hline 69 & 6 & -2 & -2 & -3 & -1 & -2 & -2 & $B_{3}$ \\
\hline 70 & 6 & -2 & -2 & -2 & -3 & -2 & -1 & $B_{4}$ \\
\hline 71 & 6 & -2 & -2 & -2 & -3 & -1 & -2 & $B_{4}$ \\
\hline 72 & 6 & -2 & -2 & -2 & -2 & -3 & -1 & $B_{5}$ \\
\hline 73 & 6 & -2 & -2 & -2 & -2 & -1 & -3 & $B_{6}$ \\
\hline 74 & 6 & -2 & -2 & -2 & -1 & -3 & -2 & $B_{5}$ \\
\hline 75 & 6 & -2 & -2 & -2 & -1 & -2 & -3 & $B_{6}$ \\
\hline 76 & 6 & -2 & -2 & -1 & -3 & -2 & -2 & $B_{4}$ \\
\hline 77 & 6 & -2 & -2 & -1 & -2 & -3 & -2 & $B_{5}$ \\
\hline 78 & 6 & -2 & -2 & -1 & -2 & -2 & -3 & $B_{6}$ \\
\hline 79 & 6 & -2 & -1 & -3 & -2 & -2 & -2 & $B_{3}$ \\
\hline 80 & 6 & -2 & -1 & -2 & -3 & -2 & -2 & $B_{4}$ \\
\hline 81 & 6 & -2 & -1 & -2 & -2 & -3 & -2 & $B_{5}$ \\
\hline 82 & 6 & -2 & -1 & -2 & -2 & -2 & -3 & $B_{6}$ \\
\hline
\end{tabular}


Table 4 continued

\begin{tabular}{llrrrrrrl}
\hline$\#$ & $L$ & $E_{1}$ & $E_{2}$ & $E_{3}$ & $E_{4}$ & $E_{5}$ & $E_{6}$ & Div. class \\
\hline $\mathbf{8 3}$ & 6 & -1 & -3 & -2 & -2 & -2 & -2 & $B_{2}$ \\
$\mathbf{8 4}$ & 6 & -1 & -2 & -3 & -2 & -2 & -2 & $B_{3}$ \\
$\mathbf{8 5}$ & 6 & -1 & -2 & -2 & -3 & -2 & -2 & $B_{4}$ \\
$\mathbf{8 6}$ & 6 & -1 & -2 & -2 & -2 & -3 & -2 & $B_{5}$ \\
$\mathbf{8 7}$ & 6 & -1 & -2 & -2 & -2 & -2 & -3 & $B_{6}$ \\
$\mathbf{8 8}$ & 5 & -2 & -2 & -2 & -2 & -2 & -1 & $B_{1}$ \\
$\mathbf{8 9}$ & 5 & -2 & -2 & -2 & -2 & -1 & -2 & $B_{1}$ \\
$\mathbf{9 0}$ & 5 & -2 & -2 & -2 & -1 & -2 & -2 & $B_{1}$ \\
$\mathbf{9 1}$ & 5 & -2 & -2 & -1 & -2 & -2 & -2 & $B_{1}$ \\
$\mathbf{9 2}$ & 5 & -2 & -1 & -2 & -2 & -2 & -2 & $B_{1}$ \\
$\mathbf{9 3}$ & 5 & -1 & -2 & -2 & -2 & -2 & -2 & $B_{2}$ \\
$\mathbf{9 4}$ & 3 & -1 & -1 & -1 & -1 & -1 & 0 & $L_{1}$ \\
$\mathbf{9 5}$ & 3 & -1 & -1 & -1 & -1 & 0 & -1 & $L_{1}$ \\
$\mathbf{9 6}$ & 3 & -1 & -1 & -1 & 0 & -1 & -1 & $L_{1}$ \\
$\mathbf{9 7}$ & 3 & -1 & -1 & 0 & -1 & -1 & -1 & $L_{1}$ \\
$\mathbf{9 8}$ & 3 & -1 & 0 & -1 & -1 & -1 & -1 & $L_{1}$ \\
$\mathbf{9 9}$ & 3 & 0 & -1 & -1 & -1 & -1 & -1 & $L_{2}$ \\
\hline
\end{tabular}

\section{Appendix B: Geometric description of the distance functions}

In this section we give a more geometric definition of the distance function, and prove its equivalence to the ones given by the formulae in Sect. 2 .

Given a variety $X$ defined over $k$ let $\widetilde{X \times X}$ be the blowup of $X \times X$ along the diagonal, with exceptional divisor $E$. Let $v$ be a place of $k$ extended to $\bar{k}$ (which we also call $v$ ), and choose a $v$-adic metric on $\mathcal{O}_{\widetilde{X \times X}}(E)$, i.e., a (non-trivial) $v$-adic norm $|\cdot|_{v}$ on each fibre of $\mathcal{O}_{\widetilde{X \times X}}(E) \otimes k_{v}$, varying continuously with the points of $(\widetilde{X \times X})\left(k_{v}\right)$ (see e.g., [3, p. 162] for a discussion of $v$-adic metrics). We fix a nonzero global section $s_{E}$ of $\mathcal{O}_{\widetilde{X \times X}}(E)$ with divisor $E$. Given points $x, y \in X(k)$ with $x \neq y$, then $(x, y)$ is a point of $X \times X$ not on the diagonal, and hence corresponds to a unique point, which we also label $(x, y)$ of $\widetilde{X \times X}$. We then define a distance function $d_{v}^{\prime}(\cdot, \cdot)$ by

$$
d_{v}^{\prime}(x, y)= \begin{cases}\left|s_{E}(x, y)\right|_{v} & \text { if } x \neq y \\ 0 & \text { if } x=y\end{cases}
$$

Different choices of $s_{E}$ differ by a scalar, and induce equivalent distance functions. Similarly, different choices of $v$-adic metrics differ multiplicatively on $\widetilde{X \times X}$ by a bounded function (see [3, p. 162, Lemma 7.1]), and again induce equivalent distance functions.

The distance functions $d_{v}(\cdot, \cdot)$ in Sect. 2 are obtained by restricting distance functions on $\mathbb{P}^{r}$ under an embedding $X \hookrightarrow \mathbb{P}_{k}^{r}$. We may also view the distance function 
$d_{v}^{\prime}(\cdot, \cdot)$ defined above as being induced from a distance function on projective space, and this will allow us to reduce the problem of showing the equivalence of the two types of distance functions to the case $X=\mathbb{P}^{r}$. To see this we note that an embedding $X \hookrightarrow \mathbb{P}_{k}^{r}$ induces an embedding $X \times X \hookrightarrow \mathbb{P}^{r} \times \mathbb{P}^{r}$. Let $\widehat{\mathbb{P}^{r} \times \mathbb{P}^{r}}$ be the blowup of $\mathbb{P}^{r} \times \mathbb{P}^{r}$ along its diagonal $\Delta_{\mathbb{P}^{r}}$. The proper transform of $X \times X$ in $\widetilde{\mathbb{P}^{r} \times \mathbb{P}^{r}}$ is $\widehat{X \times X}$. Furthermore, the exceptional divisor $E$ in $\widetilde{\mathbb{P r}^{r} \times \mathbb{P}^{r}}$ restricts to the exceptional divisor on $\widetilde{X \times X}$, and a $v$-adic metric on $\mathcal{O}_{\widetilde{\mathbb{P}^{r} \times \mathbb{P}^{r}}}(E)$ restricts to one on the corresponding line bundle on $\widehat{X \times X}$. Thus the distance function $d_{v}^{\prime}(\cdot, \cdot)$ on $\mathbb{P}^{r}$ restricts to the distance function $d_{v}^{\prime}(\cdot, \cdot)$ on $X$, and to show that $d_{v}^{\prime}(\cdot, \cdot)$ and $d_{v}(\cdot, \cdot)$ are equivalent we may assume that $X=\mathbb{P}^{r}$.

Let $\left(\left[Z_{0}: \cdots: Z_{r}\right],\left[W_{0}: \cdots: W_{r}\right]\right)$ be coordinates on $\mathbb{P}^{r} \times \mathbb{P}^{r}$. The diagonal of $\mathbb{P}^{r} \times \mathbb{P}^{r}$ is cut out by the equations $Z_{i} W_{j}-Z_{j} W_{i}=0$ for $0 \leqslant i<j \leqslant r$. Set $N=\left(\begin{array}{c}r+1 \\ 2\end{array}\right)-1$, and let $U_{i, j}, 0 \leqslant i<j \leqslant r$, be coordinates on $\mathbb{P}^{N}$ (in some chosen order). The blowup $\widehat{\mathbb{P}^{r} \times \mathbb{P}^{r}}$ is the closure in $\mathbb{P}^{r} \times \mathbb{P}^{r} \times \mathbb{P}^{N}$ of the graph of the rational map

$$
\begin{gathered}
\mathbb{P}^{r} \times \mathbb{P}^{r} \rightarrow \mathbb{P}^{N} \\
{\left[Z_{0}: \cdots: Z_{r}\right] \times\left[W_{0}: \cdots: W_{r}\right] \mapsto\left[Z_{0} W_{1}-Z_{1} W_{0}: \cdots: Z_{r-1} W_{r}-Z_{r} W_{r-1}\right] .}
\end{gathered}
$$

Among the equations cutting out $\widetilde{\mathbb{P}^{r} \times \mathbb{P}^{r}}$ in $\mathbb{P}^{r} \times \mathbb{P}^{r} \times \mathbb{P}^{N}$ are

$$
\left(Z_{i} W_{j}-Z_{j} W_{i}\right) U_{\ell m}=\left(Z_{\ell} W_{m}-Z_{m} W_{\ell}\right) U_{i j}
$$

for all pairs $(i, j),(\ell, m)$, with $0 \leqslant i<j \leqslant r$, and $0 \leqslant \ell<m \leqslant r$. These equations simply express that the functions $Z_{i} W_{j}-Z_{j} W_{i}$ were used to give the rational map to $\mathbb{P}^{N}$. On an the open subset of $\widehat{\mathbb{P}^{r} \times \mathbb{P}^{r}}$ where $U_{i j} \neq 0$ and $U_{\ell m} \neq 0$, we can rewrite this as the relation

$$
\left.\frac{Z_{i} W_{j}-Z_{j} W_{i}}{U_{i j}}\right|_{\widehat{\mathbb{P r} \times \mathbb{P}^{r}}}=\left.\frac{Z_{\ell} W_{m}-Z_{m} W_{\ell}}{U_{\ell m}}\right|_{\widehat{\mathbb{P}^{r} \times \mathbb{P}^{r}}} .
$$

Let $E$ be the exceptional divisor of the blowup. The line bundle $\mathcal{O}_{\widehat{\mathbb{P r}} \times \mathbb{P}^{r}}(E)$ is the restriction of $\mathcal{O}_{\mathbb{P}^{r} \times \mathbb{P}^{r} \times \mathbb{P}^{N}}(1,1,-1)$ to $\widehat{\mathbb{P r}^{r} \times \mathbb{P}^{r}}$. On the open set of $\widehat{\mathbb{P r}^{r} \times \mathbb{P}^{r}}$ where $U_{i j} \neq$ 0 , restricting $\left(Z_{i} W_{j}-Z_{j} W_{i}\right) / U_{i j}$ gives a section of $\left.\mathcal{O}_{\mathbb{P}^{r} \times \mathbb{P}^{r} \times \mathbb{P}^{N}}(1,1,-1)\right|_{\mathbb{P}^{r} \times \mathbb{P}^{r}}=$ $\mathcal{O}_{\overparen{\mathbb{P r}^{r} \times \mathbb{P}^{r}}}(E)$. The equation above shows that these local sections patch together to give a global section $s_{E}$ of $\mathcal{O}_{\mathbb{P r} \times \mathbb{P}^{r}}(E)$. This section has divisor $E$, and so we may use it to compute the distance. To give a $v$-adic metric on $\mathcal{O}_{\mathbb{P}^{r} \times \mathbb{P}^{r}}(E)$, we put $v$-adic metrics on $\mathcal{O}_{\mathbb{P}^{r} \times \mathbb{P}^{r} \times \mathbb{P}^{N}}(1,0,0), \mathcal{O}_{\mathbb{P}^{r} \times \mathbb{P}^{r} \times \mathbb{P}^{N}}(0,1,0)$, and $\mathcal{O}_{\mathbb{P}^{r} \times \mathbb{P}^{r} \times \mathbb{P}^{N}}(0,0,1)$, by putting an explicit $v$-adic metric on $\mathcal{O}_{\mathbb{P}^{r}}(1)$ and $\mathcal{O}_{\mathbb{P}^{N}}(1)$ as described below, and pull these back from the factors. These then give a $v$-adic metric on $\mathcal{O}_{\mathbb{P}^{r} \times \mathbb{P}^{r} \times \mathbb{P}^{N}}(1,1,-1)$ which we restrict to get a $v$-adic metric on $\mathcal{O}_{\mathbb{P}^{r} \times \mathbb{P}^{r}}(E)$. Here is our choice of $v$-adic metric on $\mathcal{O}_{\mathbb{P}^{r}}(1)$ (and similarly for $\mathcal{O}_{\mathbb{P}^{N}}(1)$ ). Let $V$ be the $k$-vector space underlying $\mathbb{P}^{r}$ so that 
$\mathbb{P}^{r}=\mathbb{P}(V)$, and $Z_{0}, \ldots, Z_{r}$ the coordinates on $\mathbb{P}^{r}$ (i.e., a chosen basis for $V^{*}$ ). Given any $\tilde{x} \in V$ and section $s \in H^{0}\left(\mathbb{P}^{r}, \mathcal{O}_{\mathbb{P}^{r}}(1)\right)=V^{*}$ we may evaluate $s(\widetilde{x})$ to get an element of $k_{v}$. Given any $x \in \mathbb{P}^{r}$ we set

$$
|s(x)|_{v}=\frac{\|s(\widetilde{x})\|_{v}}{\max _{0 \leqslant i \leqslant r}\left(\left\|Z_{i}(\widetilde{x})\right\|\right)}
$$

where $\tilde{x} \in V$ is any representative of $x$. The formula above does not depend on the choice of representative $\tilde{x}$, and so is well defined; for this reason we will use the notation $s(x)$ and $Z_{i}(x)$ in further formulae. If two sections $s$ and $s^{\prime}$ are equal at $x$, then $|s(x)|_{v}=\left|s^{\prime}(x)\right|_{v}$, and hence the formula above puts a $v$-adic metric on the fibre at $x$. This metric varies continuously with $x \in X\left(k_{v}\right)$.

Let $x=\left[x_{0}: \cdots: x_{r}\right]$ and $y=\left[y_{0}: \cdots: y_{r}\right]$ be points of $\mathbb{P}^{r}$, with $x \neq y$, so that $(x, y)$ is a point of $\mathbb{P}^{r} \times \mathbb{P}^{r}$ not on the diagonal. The corresponding point on $\widehat{\mathbb{P}^{r} \times \mathbb{P}^{r}}$ (in the coordinates of the embedding in $\left.\mathbb{P}^{r} \times \mathbb{P}^{r} \times \mathbb{P}^{N}\right)$ is $\left(\left[x_{0}: \cdots: x_{r}\right],\left[y_{0}: \cdots: y_{r}\right],\left[x_{0} y_{1}-\right.\right.$ $\left.\left.x_{1} y_{0}: \cdots: x_{r-1} y_{r}-x_{r} y_{r-1}\right]\right)$. I.e., up to independent scalars in the $Z, W$, and $U$ variables, $Z_{i}(x, y)=x_{i}, W_{j}(x, y)=y_{j}$, and $U_{i j}(x, y)=x_{i} y_{j}-x_{j} y_{i}$. Choosing any $(\ell, m)$ such that $U_{\ell m}(x, y) \neq 0$, so that $s_{E}$ can be represented near $(x, y)$ by $\left(Z_{\ell} W_{m}-Z_{m} W_{\ell}\right) / U_{\ell m}$ we compute that

$$
\begin{aligned}
d_{v}^{\prime}(x, y) & =\left|s_{E}(x, y)\right|_{v} \\
& =\frac{\max _{0 \leqslant i<j \leqslant r}\left(\left\|U_{i j}(x, y)\right\|_{v}\right)}{\max _{0 \leqslant i \leqslant r}\left(\left\|Z_{i}(x, y)\right\|_{v}\right) \max _{0 \leqslant j \leqslant r}\left(\left\|W_{j}(x, y)\right\|_{v}\right)} \\
& \cdot\left\|\frac{\left(Z_{\ell} W_{m}-Z_{m} W_{\ell}\right)(x, y)}{U_{\ell m}(x, y)}\right\|_{v} \\
& =\frac{\max _{0 \leqslant i<j \leqslant r}\left(\left\|x_{i} y_{j}-x_{j} y_{i}\right\|_{v}\right)}{\max _{0 \leqslant i \leqslant r}\left(\left\|x_{i}\right\|_{v}\right) \max _{0 \leqslant j \leqslant r}\left(\left\|y_{j}\right\|_{v}\right)} \cdot \frac{\left\|x_{\ell} y_{m}-x_{m} y_{\ell}\right\|_{v}}{\left\|x_{\ell} y_{m}-x_{m} y_{\ell}\right\|_{v}} \\
& =\frac{\max _{0 \leqslant i<j \leqslant r}\left(\left\|x_{i} y_{j}-x_{j} y_{i}\right\|_{v}\right)}{\max _{0 \leqslant i \leqslant r}\left(\left\|x_{i}\right\|_{v}\right) \max _{0 \leqslant j \leqslant r}\left(\left\|y_{j}\right\|_{v}\right)} .
\end{aligned}
$$

Here $\max \left(\left\|Z_{i}(x, y)\right\|_{v}\right), \max \left(\left\|W_{j}(x, y)\right\|_{v}\right)$, and $\max \left(\left\|U_{i j}(x, y)\right\|_{v}\right)$, come from

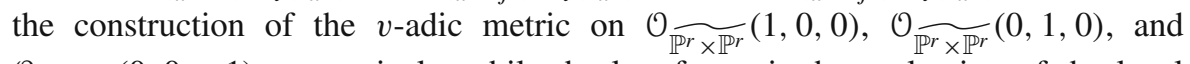
$\mathcal{O}_{\overparen{\operatorname{Pr}} \times \mathbb{P} r}(0,0,-1)$, respectively, while the last factor is the evaluation of the local representation of $s_{E}$ at $(x, y)$.

As the equation shows, for non-archimedean $v$ we have $d_{v}(x, y)=d_{v}^{\prime}(x, y)$. For archimedean $v$ we first consider the case that $k_{v}=\mathbb{C}$. The issue is then to compare

$$
d_{v}(x, y)=1-\frac{\left|\sum_{i=0}^{r} x_{i} \overline{y_{i}}\right|^{2}}{\left(\sum_{i=0}^{r}\left|x_{i}\right|^{2}\right)\left(\sum_{j=0}^{r}\left|y_{j}\right|^{2}\right)}=\frac{\sum_{0 \leqslant i<j \leqslant r}\left|x_{i} y_{j}-x_{j} y_{i}\right|^{2}}{\left(\sum_{0 \leqslant i \leqslant r}\left|x_{i}\right|^{2}\right)\left(\sum_{0 \leqslant j \leqslant r}\left|y_{j}\right|^{2}\right)},
$$

with

$$
d_{v}^{\prime}(x, y)=\frac{\max _{0 \leqslant i<j \leqslant r}\left(\left|x_{i} y_{j}-x_{j} y_{i}\right|^{2}\right)}{\max _{0 \leqslant i \leqslant r}\left(\left|x_{i}\right|^{2}\right) \max _{0 \leqslant j \leqslant r}\left(\left|y_{j}\right|^{2}\right)} .
$$


On $\mathbb{C}^{s}$ with coordinate functions $t_{1}, \ldots, t_{s}$, we have

$$
\frac{1}{s}\left(\left|t_{1}\right|^{2}+\cdots+\left|t_{s}\right|^{2}\right) \leqslant \max \left(\left|t_{1}\right|^{2}, \ldots,\left|t_{s}\right|^{2}\right) \leqslant\left|t_{1}\right|^{2}+\cdots+\left|t_{s}\right|^{2}
$$

so that $\left|t_{1}\right|^{2}+\cdots+\left|t_{s}\right|^{2}$ and $\max \left(\left|t_{1}\right|^{2}, \ldots,\left|t_{s}\right|^{2}\right)$ are equivalent functions on $\mathbb{C}^{s}$. Applying this equivalence between the max and the sum to the factors in the numerator and denominator of $d_{v}(\cdot, \cdot)$ shows that $d_{v}(\cdot, \cdot)$ and $d_{v}^{\prime}(\cdot, \cdot)$ are equivalent.

In the case that $v$ is archimedean and $k_{v}=\mathbb{R}$, the comparison is between

$$
\begin{aligned}
& d_{v}(x, y)=\left(\frac{\sum_{0 \leqslant i<j \leqslant r}\left|x_{i} y_{j}-x_{j} y_{i}\right|^{2}}{\left(\sum_{0 \leqslant i \leqslant r}\left|x_{i}\right|^{2}\right)\left(\sum_{0 \leqslant j \leqslant r}\left|y_{j}\right|^{2}\right)}\right)^{1 / 2} \text { and } \\
& d_{v}^{\prime}(x, y)=\frac{\max _{0 \leqslant i<j \leqslant r}\left(\left|x_{i} y_{j}-x_{j} y_{i}\right|\right)}{\max _{0 \leqslant i \leqslant r}\left(\left|x_{i}\right|\right) \max _{0 \leqslant j \leqslant r}\left(\left|y_{j}\right|\right)},
\end{aligned}
$$

which, after squaring the distance functions, reduces to the previous case. Thus in all cases $d_{v}(\cdot, \cdot)$ and $d_{v}^{\prime}(\cdot, \cdot)$ are equivalent distance functions.

Acknowledgments We thank Kevin Hare for useful computations performed in the initial stages of this project. We are grateful to the referees of this paper who provided many useful suggestions on the exposition and advice on strengthening one of the results. Finally, we wish to acknowledge an intellectual debt to Mike Nakamaye, who has long advocated the point of view that Seshadri constants are diophantine.

\section{References}

1. Arzhantsev, I., Derenthal, U., Hausen, J., Laface, A.: Cox Rings. Cambridge Studies in Advanced Mathematics, vol. 144. Cambridge University Press, New York (2015)

2. Bombieri, E., Gubler, W.: Heights in Diophantine Geometry. New Mathematical Monographs, vol. 4. Cambridge University Press, Cambridge (2006)

3. Cornell, G., Silverman, J.H. (eds.): Arithmetic Geometry. Springer, New York (1986)

4. Demailly, J.-P.: Singular hermitian metrics on positive line bundles. In: Hulek, K., Peternell, T., Schneider, M., Schreyer, F.-O. (eds.) Complex Algebraic Varieties. Lecture Notes in Mathematics, vol. 1507, pp. 87-104. Springer, Berlin (1992)

5. Hindry, M., Silverman, J.H.: Diophantine Geometry. Graduate Texts in Mathematics, vol. 201. Springer, New York (2000)

6. Lang, S.: Diophantine Geometry. Interscience Tracts in Pure and Applied Mathematics, vol. 11. Interscience Publishers, New York (1962)

7. Lazarsfeld, R.: Positivity in Algebraic Geometry I. Ergebnisse der Mathematik und ihrer Grenzgebiete, vol. 48. Springer, Berlin (2004)

8. Liouville, J.: Nouvelle démonstration d'un théorème sur les irrationnelles algébriques. C. R. Acad. Sci. Paris, Sér. A 18, 910-911 (1844)

9. McKinnon, D.: A conjecture on rational approximations to rational points. J. Algebraic Geom. 16(2), 257-303 (2007)

10. McKinnon, D., Roth, M.: Seshadri constants, diophantine approximation, and Roth's theorem for arbitrary varieties. Invent. Math 200(2), 513-583 (2015)

11. McKinnon, D.: Generators of subcones of the nef cone of a cubic surface, tables computed with help of the package http://www.iwr.uni-heidelberg.de/groups/comopt/software/PORTA/. Tables available at http://www.math.uwaterloo.ca/ dmckinno/Papers/cubictable.pdf

12. Serre, J.P.: Lectures on the Mordell-Weil Theorem. Aspects of Mathematics, 3rd edn. Vieweg, Braunschweig (1997) 
13. Vojta, P.: Diophantine Approximations and Value Distribution Theory. Lecture Notes in Mathematics, vol. 1239. Springer, Berlin (1987) 